\title{
Abstracts from the Conference
}

\section{NEUROPATHOLOGY. \\ NEUROGENETICS 2019}

\author{
Warsaw, 15 November, 2019
}

\begin{abstract}
ORGANIZERS
${ }^{1}$ The Team of Oncologic Neuropathology of the Committee of Neurological Sciences,

Polish Academy of Sciences

${ }^{2}$ Polish Association of Neuropathologists

${ }^{3}$ Department of Experimental and Clinical Neuropathology,

Mossakowski Medical Research Centre, Polish Academy of Sciences
\end{abstract}

\section{SCIENTIFIC PROGRAMME COMMITTEE}

\author{
Ewa Matyja \\ Ewa Iżycka-Świeszewska \\ Teresa Wierzba-Bobrowicz \\ Wiesława Grajkowska \\ Maria Łastowska \\ Milena Laure-Kamionowska \\ Dorota Dziewulska \\ Aleksandra Sejda
}





\section{TDP-43 pathology in frontotemporal lobar degeneration (FTLD) with MAPT mutation}

\author{
Albert Acewicz ${ }^{1}$, Teresa Wierzba-Bobrowicz ${ }^{1}$, \\ Sylwia Tarka ${ }^{1,2}$, Paulina Felczak ${ }^{1}$, Tomasz Stępień ${ }^{1}$, \\ Dominik Chutorański ${ }^{1}$, Mariusz Berdyński ${ }^{3}$
}

\author{
${ }^{1}$ Department of Neuropathology, Institute of Psychiatry \\ and Neurology, Warsaw, Poland \\ ${ }^{2}$ Department of Forensic Medicine, Warsaw Medical University, \\ Poland \\ ${ }^{3}$ Department of Neurodegenerative Disorders and Neurogenetic \\ Unit, Mossakowski Medical Research Institute, Polish Academy \\ of Sciences, Warsaw, Poland
}

Introduction: Frontotemporal lobar degeneration (FTLD) describes a spectrum of neurodegenerative disorders with heterogeneous clinical presentation, pathology and genetic abnormalities, which result in atrophy of the frontal and temporal lobes. Among hereditary forms of FTLD mutation in microtubule-associated protein tau gene (MAPT) has been identified as major genetic factor in the pathogenesis. Although, Tau pathology is considered to be main cause of neurodegeneration in FTLD with MAPT mutation, some reports indicate that TDP-43 may play a role in the pathogenesis.

Material and methods: We investigated brains samples from 6 patients with FTLD (4 sporadic vs. 2 genetic cases with mutation in MAPT) employing routine histological examination and immunohistochemistry for marker proteins Tau and TDP-43. Immunostained sections were assessed for the appearance, distribution and intensity of staining in neurons of the frontal lobes and temporal lobes including hippocampus.

Results: In all cases routine histology examination showed neocortical neuronal loss in the frontal and temporal lobes, accompanied by moderate spongiosis and astrogliosis. Tau-immunostaining revealed abundant deposists in the both frontal and temporal cortex in the form of neurites, neurofibrillary tangles (NFTs), neuropil threads (NTs), coiled bodies, Pick bodies, ballooned neurons, grain-like deposits. Similarly, in both sporadic and hereditary form of FTLD we observed abundant nuclear and single cytoplasmic TDP-43 aggregates in frontal and temporal lobe neurons with no significant differences in distribution and intensity between cases.

Conclusions: We confirm that Tau seems to play crucial and TDP-43 additional role in the pathogenesis of FTLD with MAPT mutation. Although, more studies on larger group of cases are needed to reveal exact relationships between proteins involved in FTLD pathogenesis.

\section{Activity of phrenic and hypoglossal respiratory neurons and peripheral dopaminergic transmission in 6-OHDA lesioned rats}

\author{
Kryspin Andrzejewski, Monika Jampolska, \\ Katarzyna Kaczyńska
}

Department of Respiration Physiology, Mossakowski Medical Research Centre, Polish Academy of Sciences, Warsaw, Poland

Respiratory disturbances present in Parkinson's disease (PD) are not well understood. In this study intracerebroventricular injection of 6-hydroxydopamine (6-OHDA) into the third brain ventricle was performed to model PD. The main goal of the present experiments was to compare phrenic (PHR) and hypoglossal (HG) nerve activity during $8 \%$ hypoxia in 6-OHDA lesioned and sham operated male Wistar rats pretreated with desipramine.

In anesthetized, paralyzed, artificially ventilated rats, PHR and HG nerve activities: frequency, amplitude, minute activity, pre-inspiratory, inspiratory, expiratory time and total time of respiratory cycle were computed in normoxic and hypoxic conditions.

In second group of an anesthetized and spontaneously breathing rats changes in ventilatory parameters in normoxia were analyzed after peripheral dopaminergic $D_{2}$ receptors arousal by dopamine (DA) and blockade by domperidone (DOM) to investigate abnormalities in dopaminergic transmission in peripheral carotid body chemoreceptors in PD model.

Amplitude and minute activity of the PHR and HG nerves in lesioned rats exhibited significantly higher values during hypoxic ventilatory response. Analysis of ratio of pre-inspiratory time of HG nerve to the total length of the respiratory cycle displayed significant increase during air breathing and decrease while recovery from hypoxia. Ratio of pre-inspiratory amplitude to the inspiratory peak amplitude of HG nerve in 6-OHDA rats was significantly decreased in normoxia and during post-hypoxic recovery.

In 6-OHDA treated rats normoxic respiratory tidal volume $\left(V_{T}\right)$ was augmented while the frequency of breathing showed insignificant decline (f). Opposing changes in $V_{T}$ and $f$ had no influence on changes in control minute ventilation $\left(V_{E}\right)$.

Dopamine intravenous injection induced immediate depression of all respiratory parameters in neurotoxin and vehicle treated animals. Domperidone intravenous treatment caused similar increases in $\mathrm{V}_{\mathrm{T}}$ and $\mathrm{f}$ resulting in $\mathrm{V}_{\mathrm{E}}$ augmentation in sham and 6-OHDA rats.

We concluded that changes in the pre-inspiratory hypoglossal nerve activity responsible for maintaining patency of the upper airway and altered activity of HG and 
PHR nerves in lesioned animals, suggests disturbances in the central respiratory areas controlling both nerves. Lack of changes after DA and DOM treatment between both groups reject hypothesis about changes in dopaminergic transmission in the carotid body chemoreceptors in PD.

\section{The tripeptidyl peptidase 1 (TPP1) deficiency in 36-year-old patient with cerebellar- extrapyramidal syndrome and dilated cardiomyopathy}

\author{
Małgorzata Bednarska-Makaruk ${ }^{1}$, Rafał Płoski ${ }^{2}$, \\ Grażyna Truszkowska ${ }^{3}$, Agnieszka Ługowska ${ }^{1}$, \\ Maciej Pronicki ${ }^{4}$, Eliza Lewandowska ${ }^{5}$, \\ Aleksandra Podlecka-Piętowska ${ }^{6}$, Martyna Sitek ${ }^{6}$, \\ Zofia T. Bilińska ${ }^{7}$, Przemysław Leszek ${ }^{8}$
}

${ }^{1}$ Department of Genetics, Institute of Psychiatry and Neurology Warsaw, Poland

2Department of Medical Genetics, Medical University of Warsaw, Poland

${ }^{3}$ Laboratory of Molecular Biology, Institute of Cardiology, Warsaw, Poland

${ }^{4}$ Department of Pathology, The Children's Memorial Health Institute, Warsaw, Poland

${ }^{5}$ Department of Neuropathology, Institute of Psychiatry and Neurology, Warsaw, Poland

${ }^{6}$ Department of Neurology, Medical University of Warsaw, Poland ${ }^{7}$ Unit for Screening Studies in Inherited Cardiovascular Diseases, Institute of Cardiology, Warsaw, Poland

${ }^{8}$ Department of Heart Failure and Transplantology, Institute of Cardiology, Warsaw, Poland

We report a 36-years old man presenting with cerebellar-extrapyramidal syndrome as well as the severe heart failure because of dilated cardiomyopathy of unknown origin. Family history was negative and there was no consanguinity in the parents. Dysarthria and cardiac arrhythmia began at early childhood (4 yr.). Brain MRI (age 28) demonstrated severe cerebellar atrophy. Neurological examination and psychological assessment at the age 32 revealed dysarthria, ataxia, dystonia and tremor of the right hand and decreased mental acuity. VEP examination revealed bilateral slowed neural conduction in the visual pathways. Progression of the neurological symptoms is slow.

At the age of 33 patient underwent successful cardiac transplantation because of severe dilated cardiomyopathy. In light microscopy the biopsy specimens obtained from explanted heart showed severe myocyte hypertrophy with perinuclear vacuolization with inclusions suggestive of an underlying storage disease. Fabry disease was excluded by enzymatic analysis.
Next generation sequencing (NGS) was performed to target protein coding sequences in the human genome for potential disease-causing variants with the use of TruSight One sequencing panel. NGS revealed compound heterozygosity for two novel missense mutations in the TPP1 gene inherited from both parents as well as hemizygous novel missense mutation in the $A B C D 1$ gene inherited from mother, both potentially pathogenic. TPP1, encoding the tripeptidylpeptidase 1 enzyme, is known as the causative gene for late infantile neuronal ceroid lipofuscinosis 2 (CLN2). $A B C D 1$ gene mutations are responsible for $\mathrm{X}$-linked adrenoleukodystrophy/adrenomyeloneuropathy.

Further studies revealed normal VLCFA levels both in patient and his mother, thus excluding $A B C D 1$ mutation as the pathogenic one. Enzymatic analysis in blood leukocytes showed significantly reduced TPP1 activity (8.8 $\mathrm{nmol} / \mathrm{mg}$ protein/hr; reference range: $54 \pm 18.2$ ). The explanted heart tissue was also evaluated by transmission electron microscopy. This revealed absence of lipofuscin accumulation, as well as of ultrastructural curvilinear profiles, fingerprint bodies and granular osmiophilic deposits (GRODs) in lysosomes characteristic of neuronal ceroid lipofuscinosis.

Sun et al. (2013) identified TPP1 as the causative gene for autosomal recessive spinocerebellar ataxia type 7 (SCAR7). It was suggested that loss of function variants abolishing TPP1 enzyme activity lead to CLN2 disease, whereas variants that diminish TPP1 enzyme activity lead to slowly progressive SCAR7.

This case report highlights the power of next-generation sequencing (NGS) in diagnosing rare and atypical diseases.

\section{Genetic variants of CYP2C9, CYP2C19, CYP3A4 among children of Ukraine suffering from drug-resistant epilepsy}

\section{Yuriy Boiko ${ }^{1}$, Yevhen Tantsura ${ }^{2}$}

${ }^{1}$ Odessa State Medical University, Odessa, Ukraine ${ }^{2}$ V.N. Karazin Kharkiv National University, Kharkiv, Ukraine

The effectiveness and safety of the treatment of epilepsy is influenced by many factors, including the activity of enzymes of the cytochrome P450 system. The main isoenzymes involved in the metabolism of anti-epileptic drugs (AED) - CYP2C9, CYP2C19, CYP3A4 are encoded by the corresponding genes. The frequency of occurrence of enzyme polymorphisms, the participation and the role of each of them in the processes of AED metabolism and the formation of drug-resistant (Anderson GD, 2008; Lopez-Garcia M et al., 2014). 
Aim of the study: To study the frequency of genetic polymorphisms - CYP2C9, CYP2C19, CYP3A4 in children with drug-resistant epilepsy and to determine their potential role, as well as the role of other factors in the development of pharmacological resistance.

Material and methods: Genotyping of CYP2C9 * 1 , CYP2C9 * 2, CYP2C9 * 3, CYP2C19 * 1, CYP2C19 * 2, CYP3A4 * $1 \mathrm{~A}, \mathrm{CYP} 3 \mathrm{~A} 4{ }^{*} 1 \mathrm{~B}$ by the method of allele-specific $P C R(P C R)$ was performed in 83 children and adolescents ( $M-49, F-34)$, residents of Ukraine, suffering from drug-resistant epilepsy.

Results: Alleles of genes that provide the slowing down of AED metabolism - CYP2C19 * 2 in 39.76\%; CYP2C9 * 2 and CYP2C9 * 3 for $20.48 \%$; CYP3A4 * 1 B at $12.05 \%$; a combination of these polymorphisms was found in $10.84 \%$ of children suffering drug-resistant epilepsy, which was significantly more frequent than in population studies in Ukraine and other European countries $(p<0.05)$. A clear connection between the presence of "slow" alleles and AED tolerability, the occurrence of side effects was revealed.

In our experimental model of drug-resistant epilepsy, chronic administration of the inhibitor (Sultiame) and activator (Carbamazepine) of the enzyme system of cytochrome P450 was used against the background of the epileptic status caused by multiple corneal kindling. The development of resistance to the anticonvulsant action of Carbamazepine and Lamotrigine has been shown with prior chronic administration of Carbamazepine. Prior chronic administration of Sultiame increased the anticonvulsant effect of Lamotrigine.

Summary: The results of genetic and experimental studies allow us to predict the response to the use of various AEDs, select therapy individually, increase the effectiveness and safety of treatment.

\section{GATA4 immunoexpression/methylation is associated with TP53 mutations, but not with prognosis in primary glioblastoma patients}

Marcin Braun ${ }^{1}$, Berenika Trąbska-Kluch², Sylwia Paszek ${ }^{3,4}$, Izabela Zawlik ${ }^{3,4}$, Dorota Jesionek-Kupnicka ${ }^{1}$

${ }^{1}$ Department of Pathology, Chair of Oncology, Medical University of Lodz, Poland

${ }^{2}$ Department of Radiotherapy, Medical University of Lodz, Poland ${ }^{3}$ Department of Genetics, Chair of Molecular Medicine, Faculty of Medicine, University of Rzeszow, Poland

${ }^{4}$ Laboratory of Molecular Biology, Centre for Innovative Research in Medical and Natural Sciences, University of Rzeszow, Poland
Introduction: GATA4 is a tumour suppressor gene, which loss of expression by methylation was showed as an important genetic occurrence in glioblastoma pathogenesis. Lately, inactivation of GATA4 has been linked to poor response to alkylating drugs and thus with a shorter overall survival among glioblastoma patients. Here, we investigated the prognostic role of GATA4 methylation and protein expression in the context of TP53 alterations in primary glioblastoma patients.

Material and methods: Formalin-fixed paraffin-embedded tumoral samples of 45 patients (67.7\% males) with histopathologically diagnosed glioblastoma between 2006 and 2011 were examined for: GATA4 and TP53 promoter methylation with methylation-specific $P C R$, for GATA4 and TP53 immunoexpression with immunohistochemistry and TP53 mutations with long-range PCR. The genetic alterations were statistically compared with standard clinico-pathological features and overall survival of recruited patients.

Results: Expression of GATA4 protein was detected in $20(57.1 \%)$ patients and was not associated with GATA4 methylation (found in 9 (25.7\%) of patients; $p=0.7002$ ). TP53 protein overexpression was found in 11 (35.5\%), TP53 mutations in 6 (19.4\%) and TP53 methylation in 3 (11.5\%) patients. Patients with preserved expression of GATA4 protein were more frequently affected by TP53 mutation comparing to patients with loss of GATA4 protein expression (40.0\% vs. $0.0 \% ; p=0.0068)$. There were no significant associations between GATA4 immunoexpression/methylation and both age at diagnosis and overall survival of recruited patients ( $p>0.05$ for all comparisons).

Conclusions: Loss of GATA4 protein expression seems to be only partially linked to GATA4 methylation, and is less common in glioblastoma patients with TP53 mutations. There was no clear prognostic impact of GATA4 alterations in the studied glioblastoma patients.

\section{Alterations of transcription of genes coding sirtuins and proteins involved in mitochondrial dynamics in amyloid $\beta$ toxicity in experimental model of Alzheimer's disease}

Magdalena Cieślik ${ }^{1}$, Grzegorz A. Czapski ${ }^{1}$, Sylwia Wójtowicz ${ }^{1}$, Iga Wieczorek ${ }^{2}$, Przemysław L. Wencel ${ }^{2}$, Robert P. Strosznajder ${ }^{2}$, Vivian Jaber ${ }^{3}$, Walter J. Lukiw ${ }^{3,4}$, Joanna B. Strosznajder ${ }^{1}$

${ }^{1}$ Department of Cellular Signalling, Mossakowski Medical Centre, Polish Academy of Sciences, Warsaw, Poland,

${ }^{2}$ Laboratory of Preclinical Research and Environmental Agents Department of Neurosurgery, Mossakowski Medical Centre, Polish Academy of Sciences, Warsaw, Poland 
${ }^{3}$ Department of Genetics, Louisiana State University Health Sciences Center, New Orleans, USA

${ }^{4}$ LSU Neuroscience Center and Departments of Neurology and Ophthalmology, Louisiana State University Health Sciences Center, New Orleans, USA

A growing body of evidence indicates that mitochondrial dysfunction is one of the earliest alterations responsible for progression of Alzheimer's disease (AD). Mitochondria are the main source of reactive oxygen species (ROS) but also the main target of ROS. Amyloid $\beta(A \beta)$, which contributes to neuronal degeneration and synaptic loss in $A D$, accelerates ROS generation and possibly triggers a 'vicious cycle': ROS - mitochondrial impairment - ROS. Increasing evidence indicates that sirtuins have neuroprotective effects in regulating oxidative stress and metabolism. The mechanisms by which $A \beta$ oligomers affect mitochondria as well as the role of sirtuins in AD-related hypometabolism remain not entirely understood. The aim our study was to analyse the impact of exogenous $A \beta_{1-42}$ oligomers $(A \beta O)$ and endogenously liberated $A \beta$ peptides on transcription of genes for sirtuins and mitochondrial dynamics -related proteins in cell lines (neuronal SH-SY5Y and microglial BV2) and in brain cortex of transgenic $A D$ (Tg-AD) mice.

Our results demonstrated significant $A \beta O$-evoked changes in transcription of genes in SH-SY5Y cells, where $\mathrm{A} \beta \mathrm{O}$ enhanced expression of Sod1 and Cat, attenuated Sirt5 and Sod2. In BV2 line, A $\beta O$ increased the level of mRNA for Sod2 and Dnm11, decreased for Sirt1, Sirt3, Gpx4 and $M f n 2$. A $\beta O$ enhanced free radical level and impaired mitochondrial membrane potential only in SH-SY5Y cells, but reduced viability of both cell types. Activators of Sirt1 more efficiently enhanced viability of SH-SY5Y than BV2 affected by $A \beta O$. Analysis of brain cortex of Tg-AD mice confirmed significant downregulation of Sirt1 and $M f n 1$ and upregulation of Dnm1l. In human AD brain, changes of microRNA pattern (miRNA-9, miRNA-34a, miRNA-146a and miRNA-155) seem to be responsible for decrease in Sirt1 expression.

Overall, our results demonstrated a diverse response of neuronal and microglial cells to $A \beta O$ toxicity. Alterations of genes encoding Sirt1 and proteins involved in mitochondrial dynamics suggest that modulation of Sirt1 and Drp1, including miRNA strategy, may be crucial for improvement of $A D$ therapy.

This work was supported by Statute Theme No 5, by Grant NCN-2014/2015/B/NZ3/01049 and grant to the LSU Eye Center from Research to Prevent Blindness (RPB); the Louisiana Biotechnology Research Network (LBRN) and NIH grants NEI EY006311, NIA AG18031 and NIA AG038834 (WJL).

\section{Microangiopathy in familial hemiplegic migraine with CACNA1A mutation}

\author{
Dorota Dziewulska ${ }^{1,2}$, Biruta Kierdaszuk ${ }^{1}$
}

${ }^{1}$ Department of Neurology, Medical University of Warsaw, Poland ${ }^{2}$ Department of Experimental and Clinical Neuropathology, Mossakowski Medical Research Institute, PAS, Warsaw, Poland

Familial hemiplegic migraine type 1 (FHM1) is a rare autosomal dominant disease caused by mutations in the CACNA1A gene encoding CaV2.1 calcium channels. Mutations affect density and biophysical properties of the channels.

We describe patients from Polish family with FHM1 and CACNA1A gene mutation, whose 18 members in four generations suffered from migraine. In 5 of them, brain MRI scans revealed hyperintense changes in the cerebral white matter. Due to the primary suspicion of CADASIL, in 2 of them, skin-muscle biopsies and genetic tests were performed in the interictal period of the disease.

Ultrastructural examination of biopsy samples taken from an 8-year-old boy and his 38-year-old father revealed similar pathological changes in microvessels resembling oncosis. In affected vessels, endothelial cells and myocytes/ pericytes were enlarged. Their cytoplasm was clear and contained an expanded endoplasmic reticulum, enlarged mitochondria and numerous intracellular vacuolar structures. Swollen endothelial cells often constricted capillary lumen.

The morphological changes described for the first time in FHM1 suggest that the disease is not only a functional disorder but also structural microangiopathy. The hyperintensive changes in brain MRI images seen in our patients may be due to microcirculation disturbances and damage to the cerebral white matter, secondary to structural changes.

\section{Ultrastructural images of siblings microvessels with a mutation in the NOTCH3 gene}

\section{Paulina Felczak ${ }^{1}$, Agnieszka Cudna ${ }^{2}$, Teresa Wierzba-Bobrowicz ${ }^{1}$, Tomasz Stepien ${ }^{1}$, Sylwia Tarka ${ }^{3}$, Albert Acewicz ${ }^{1}$}

${ }^{1}$ Department of Neuropathology, Institute of Psychiatry and Neurology, Warsaw, Poland

2Department of Genetics, Institute of Psychiatry and Neurology, Warsaw, Poland

${ }^{3}$ Department of Forensic Medicine, Warsaw Medical University, Poland

Mutations in the NOTCH 3 gene cause Cadasil syndrome (cerebral autosomal dominant arteriopathy with subcortical infarcts and leukoencephalopathy). Cadasil exhibits a wide spectrum of mutations, but their greatest 
severity is described in exons 3 and 4. CADASIL is a hereditary systemic vascular disorder, and granular osmophilic material (GOM) is an ultrastructural marker of this disease.

The ultrastructure of small blood vessels of two brothers and sister was compared with genetically confirmed CADASIL - mutation in exon 4 of the NOTCH 3 gene. Biological material was obtained from skin and muscle biopsies.

At the subcellular level, GOM deposits were observed in the basement membranes of capillaries and arterioles, at pericyte membranes and in the vicinity of smooth myocytes. GOMs were present in the vessel walls in all three examined cases, showing only variation in size, quantity and structure. The greatest intensification of changes was observed in the first brother, where in addition to GOMs, very thick basement membranes and degenerated cells of the vascular walls as well as necrotic vessels were found. In the second brother, the severity of vascular damage was slightly lower, while in the sister the highest number of normal vessels was observed.

There was no correlation between the age of patients and the severity of pathological changes in microvessels.

\section{Influence of oxygen-glucose deprivation on glial cell interactions}

\section{Justyna Gargas, Justyna Janowska, Malgorzata Ziemka-Nalecz, Karolina Ziabska, Joanna Sypecka}

NeuroRepair Department, Mossakowski Medical Research Centre, Polish Academy of Sciences, Warsaw, Poland

Hypoxic-ischemic episode (HI), associated with a temporal deficiency in oxygen and trophic supply, is known to trigger diversified response of cells forming the nervous tissue. Altered tissue homeostasis modulate functioning of glial cells, including differentiation of oligodendrocytes and their ability for myelinogenesis, activation of astrocytes or polarization of microglia. It is supposed that either initiation of endogenous reparative mechanisms or development of neurodegenerative processes after $\mathrm{HI}$ injury strongly depends on mutual interaction of glial cells. To verify this hypothesis, we performed studies aimed at determining the $\mathrm{HI}$ impact on the selected functions of glial cells, like their paracrine activity or migratory potential. In our research, we used an in vitro model of perinatal asphyxia. In this model, the primary mixed glial cultures were established from the isolated hemispheres of Wistar rats (one- to two-day old) and were subsequently used for derivation of purified fractions of glial cells. Next, the co-cultures of oligodendrocyte progenitor cells (OPCs) with either microglia or astrocytes were established. The cells were subjected to the oxygen-glucose deprivation procedure (OGD) for 50 minutes and then cultured for 24 h, 72 h or 5 days. The migratory potential of glial cells was checked by application of scratch assay. The cells and media were then collected for biochemical analyses or the cells were stained for the purpose of immunocytochemical analyses. As revealed by the obtained results, the pro-inflammatory phenotype of microglia prevails $24 \mathrm{~h}$ after $\mathrm{HI}$ episode. As for OPCs, the first stages of their differentiation were detected already at $24 \mathrm{~h}$ after $\mathrm{HI}$, while in OGD subjected oligodendrocytes the differentiation process was hampered. Additionally, the studies shown an activation of astrocytes, as deduced from the changed secretion of interleukin (IL)-10 and IL-1 $\beta$ to the culture medium. To sum up, disruption of the flow of oxygen and glucose leads to impairment in OPCs development and exerts a significant impact on the glial paracrine activity in context of secretion of the selected interleukins. Modulation of oligodendroglial differentiation, polarization of microglia and activation of astrocytes might altogether contribute to the processes initiated after hypoxic-ischemic event and resulting either in neuroregeneration or initiation of neurodegenerative processes.

Supported by NCN (National Science Centre, Poland) 2014/15/B/NZ4/01875 and ESF, POWR.03.02.0000-I028/17-00. Picture recording was performed in Laboratory of Advanced Microscopy Techniques, Mossakowski Medical Research Centre, Polish Academy of Sciences.

\section{'Digital Brain' resources presentation - based on selected neurological disease}

\author{
Maciej P Golan ${ }^{1}$, Tomasz Stępień ${ }^{1}$, \\ Teresa Wierzba-Bobrowicz ${ }^{1}$, \\ Anna Mietelska-Porowska ${ }^{1,2}$, Albert Acewicz ${ }^{1}$, \\ Dominik Churotański ${ }^{1}$, Sylwia Tarka ${ }^{1,3}$, Natalia Chmura ${ }^{1}$, \\ Ludomir Z. Niewiadomski ${ }^{1}$ \\ ${ }^{1}$ Department of Neuropathology, Institute of Psychiatry and \\ Neurology, Warsaw, Poland \\ ${ }^{2}$ Neurobiology Center, Nencki Institute of Experimental Biology \\ PAS, Warsaw, Poland \\ ${ }^{3}$ Department of Forensic Medicine, Medical University \\ of Warsaw, Poland
}

Human Brain Banks are one of the most essential parts in the research in the fields of neuroanatomy, neuropathology and neurobiology. The need of the high quality banked tissue is rising over the years, due to increasing number of researchers, studying human diseases or bio- 
logical processes. It is also driven by a growing need to verify the experiments' results from the other models.

As an answer to this need, Department of Neuropathology (IPiN, Warsaw) took on the task, of creating The First Polish Brain Bank. The Bank resources are based on half century long Department's activity and tissue collection. Up to date, there are more than 5200 samples, stored in the Department, which constitute the core of the Brain Bank. The collection includes: vascular disorders, infectious diseases, demyelinating diseases, metabolic disorders, neurodegenerative diseases and others. It includes also the control tissue. All of the cases are stored as a: formalin fixed sections, paraffin - embedded tissue, and glass slides. For each of the cases medical history is available.

In 'Digital Brain' cases will be presented as tissue material photographs (each slice documented bilaterally, to present structures), paraffin blocks' photographs (with detailed description of brain's structures) and scans of the glass slides.

As an answer for a need to a need to make a Bank useful for a scientific community special Data Base was designed. It allows users to explore resources of the Brain Bank and find necessary cases based on: clinical diagnosis, accompanying diseases, neuropathological findings, sex, age, additional diagnostics protocol, brain structures preserved in paraffin blocks and stainings. The user will be able to check collected materials, check the neuropathological findings and obtain basic donor's information. The Data Base will also allow further expansion of the collection.

Here we presented an exemplary case of SSPE from our database We also show a principle of operation for a First Polish Brain Bank browser and data base.

Acknowledgements: The First Polish Brain was created with a support of European Union Funds (Cyfrowa Polska) "Digital Brain - cyfrowe zasoby Instytutu Psychiatrii i Neurologii w Warszawie" - POPC 02.03.01-00.0042/18.

\section{A rare and misleading presentation of a primary glial tumour. The usefulness of molecular profiling, even in "obvious" cases}

\section{Frank R. W. van de Goot}

Symbiant Pathology Expert Centre, NWZ Location Alkmaar, Alkmaar, the Netherlands

We report the case of a 57 years old, male patient. The patient was previously diagnosed with an small cell neuro-endocrine carcinoma of the lung for which he received treatment. The patient encountered sudden collapse with a short epileptic episode.
During routine scanning (CT/MRI brain) a circumscript lesion was found in the right frontal cerebral lobe. Biopsy revealed a small cell neuro-endocrine tumour. Immunohistochemistry revealed positivity for Synaptophysin, chromogranin, CD56 and TTF-1. Ki67 was highly expressed. Pan keratin was positive as well. The immune profiling was similar to the previously diagnosed lung process.

As expected GFAP was negative as well. Due to standard procedures molecular profiling was nevertheless performed. This, leading undoubtedly to the conclusion that this was not a metastatic process from the lung but a strongly neuro-endocrine differentiated glioblastoma mimicking metastasis of a small cell lung carcinoma in a patient known with such a tumour.

This case is illustrative for the use of molecular profiling in brain tumours, even when it appears to be an "obvious" diagnosis.

\section{Molecular markers in diagnosis of paediatric brain tumours}

\section{Wiesława Grajkowska}

Department of Pathology, The Children's Memorial Health Institute, Warsaw, Poland

The currently 2016 WHO classification of CNS tumours requires assessment of molecular markers parallely to histopathology. The paediatric glioma with p.(K28M) mutation in the histone $\mathrm{H} 3$ gene $\mathrm{H} 3 \mathrm{~F} 3 \mathrm{~A}$, or less commonly in the related HISTIH3B gene, with a diffuse growth pattern, and a midline location (e.g., thalamus, brain stem, and spinal cord) is termed as diffuse midline glioma, H3 K27M-mutant. Ependymoma RELA fusion-positive is a molecular variant of supratentorial localized ependymoma in children. This subtype of ependymoma is associated with poor outcome. The L1CAM expression is a potential immuno-histochemical surrogate for this variant. Within embryonal tumors the best molecularly described is medulloblastoma. The main histological variants of medulloblastoma are: classic, desmoplastic/nodular, with extensive nodularity, large cell/anaplastic. The genetically defined four variants of medulloblastoma are: WNT-activated, SHH-activated, and the numerically designated "group 3" and "group 4" medulloblastoma. Additionally in SHH subgroup the presence of TP53 mutation has prognostic value. In the 2016 CNS WHO, the presence of C19MC amplification results in a diagnosis of embryonal tumor with multilayered rosettes (ETMR), C19MC-altered. The diagnosis of atypical teratoid/rhabdoid tumor (AT/ RT) requires confirmation of the characteristic molecular 
defects. AT/RT is now defined by alterations of either IN/1 or, very rarely, $B R G 1$. In case if a tumor has histological features of AT/RT but does not show either of the diagnostic genetic alterations, only a descriptive diagnosis of CNS embryonal tumour with rhabdoid features is available. The tumours previously designated as CNS PNETs should to be described as CNS embryonal tumors, NOS. It is hoped that identification of new molecular markers in paediatric brain tumours could lead to more precise classification and allow for improved and tailoring of patient therapy.

\section{Hypoglossal and phrenic nerves activity in reserpine model of Parkinsonism}

\author{
Monika Jampolska, Kryspin Andrzejewski, \\ Katarzyna Kaczyńska
}

Department of Respiration Physiology, Mossakowski Medical Research Centre, Polish Academy of Sciences, Warsaw

Parkinson's disease (PD) is directly related to central dopaminergic failure. Additional neurotransmission disturbances associated with PD are deficits in two other amine systems involved in the regulation of breathing, i.e. noradrenergic and serotoninergic. The underlying mechanisms of respiratory disorders present in PD are unclear. Therefore to study the activity of the main respiratory nerves: the phrenic (PHR) and the hypoglossal nerve (HG) we choose reserpine model of parkinsonism, characterized by substantial depletion of dopamine, noradrenaline and serotonin. We tested the activity of isolated nerves using bipolar electrodes on anesthetized, paralyzed and vagotomized adult Wistar rats (16 male). The procedure includes recording the amplitude and frequency of discharges in normoxia, acute hypoxia $\left(8 \% \mathrm{O}_{2}\right.$ in $\left.\mathrm{N}_{2}\right)$ and during recovery after apnea. The basal values of $\mathrm{HG}$ amplitude (A HG) and the ratio of the A pre-inspiratory $H G$ activity to $A H G$ were reduced respectively by $48 \%$ and $43 \%$ after reserpine. In the hypoxic peak A pre HG was reduced by $42 \%$ after reserpine in compare to sham group. There was no statistical difference in A HG in the hypoxic peak between both groups but in recovered post apneic breathing the A HG was much higher in reserpine rats. We observed a tendency in reserpine rats to reduced inspiratory time, with the total respiratory time unchanged compared to sham. There was no significant differences in the frequency of nerve discharges, the amplitude and minute activity of the PHR. Concluding, a deficiency in dopamine, noradrenaline and serotonin results in a reduction in amplitude of preand inspiratory HG nerve activity, which suggests altered brainstem control of the nerve responsible for maintaining upper airway patency. A high level of A HG in the reversion after apnea confirms the role of DA in the depressive phase of hypoxia.

\section{Interrelation of nootropic potential with NCAM's levels in rat hippocampus with administration of s-adenosyl-l-metionin and pre/probiotics associated with drug-induced liver injury}

\author{
Yliia Kharchenko ${ }^{1}$, Volodymyr Zhyliuk ${ }^{1}$, \\ Stefan G Pierzynowski², Jaroslav Wolinski ${ }^{3}$ \\ ${ }^{1}$ State Institution "Dnipropetrovsk Medical Academy \\ of the Ministry of Health of Ukraine", Dnipro, Ukraine \\ ${ }^{2}$ Lund University Department of Biology, Lund, Sweden \\ ${ }^{3}$ Kielanowski Institute of Animal Physiology and Nutrition, \\ Jablonna, Poland
}

Relevance: The combined use of isoniazid and rifampicin is accompanied by the effect of co-potentiating the toxic properties of these agents, which increases the risk of significant damage to the liver. During the last decade, a large amount of information on the influence of antimicrobial agents on the central nervous system (CNS) has been gathered. It is possible that the influence of rifampicin and isoniazid on the organism and, in particular, the brain, may be mediated not only by their hepatotoxicity, but also by the ability to cause a disturbance of the balance of the intestinal microflora, which has a negative effect on the course of encephalopathy in toxic lesions of the liver.

Aim of the study: To study the effect of SadenosylLmethionine, pre/probiotics, and their combined use on mnestic processes and levels of adhesive proteins in the rat hippocampus with prolonged administration of rifampicin and isoniazid.

Material and methods: Studies were performed on 68 Wistar male rats weighing 180-220 g. An experimental model of DILI was formed by using repeated gastric administration of isoniazid and rifampicin at doses of $50 \mathrm{mg} / \mathrm{kg}$ and $86 \mathrm{mg} / \mathrm{kg}$ body weight for 28 days. Animals were divided into five groups ( $n=8$ each). I - intact, II control (DILI). The rats of group III were intramuscularly injected with SadenosylLmethionine at a dose of $35 \mathrm{mg} /$ $\mathrm{kg}$ an hour before the administration of tuberculostatic drugs during the last 14 days of the experiment. The rats of group IV received a combination therapy that combined a prebiotic Lactulose at a dose of $2680 \mathrm{mg} / \mathrm{kg}$ and a probiotic containing 4 billion active cells (CFU): Lactobacillus acidophilus, Lactobacillus rhamnosus, Streptococcus ther- 
mophilus subspru, Lactobacillus subspru. Bulgaricus at a dose of $1 \mathrm{CFU} / \mathrm{kg}$. In group $\mathrm{V}$ triple combined pharmacotherapy was carried out with the use of drugs used in the III and IV groups in appropriate doses. Nootropic activity was evaluated in the test of the conditioned response of passive avoidance with administration of scopolamine. To assess the effect of scopolamine on the learning processes, the values of animal groups (intact, $n=10$; DILI, $n=18$ ), which previously did not receive this drug, were used. The quantitative content of NCAM was defined by an ELISA inhibitory method using monoclonal antibodies against NCAM (Abcam, England) and, respectively, purified protein as a standard (Abnovo, USA) in the cytosolic fraction of the hippocampus homogenate. The obtained digital data was processed by the method of variation statistics using the statistical analysis program StatPlus, AnalystSoft Inc., version 6 (see http://www.analystsoft.com/ru/).

Results: The results of experimental studies suggest that S-adenosyl-L-methionine improved learning processes with administration of scopolamine, which was proved by a significant increase in the level of LP by 3.14 times $(p=0.027)$ in comparison with the group of animals from DILI, and the number of animals with acquired skill was $62.5 \%(p=0.007)$. At the same time with administration of this drug, an increase by $38.7 \%(p=0.004)$ of cytoplasmic NCAM forms in the hippocampus was observed, which probably had a compensatory character. It is significant that the correction of the intestinal microbiota state can also have a positive effect on mnemonic functions in conditions of hepatitis with prolonged administration of tuberculostatics, however, the Lactulose/Yoghurt combination is inferior to SadenosylLmethionine in its nootropic potential, despite the fact that the NCAM level in the hippocampus increased by $39.6 \%(p=0.004)$ compared with the group of animals from DILI.

Conclusions: Combined use of prebiotic/probiotic along with SadenosylLmethionine was not accompanied by an increase in nootropic activity. The obtained data is primarily an experimental justification for the use of SadenosylLmethionine, as well as pre/probiotics, as promising means of correcting cognitive impairment in drug-induced hepatitis, and is also the subject of further research related to the possibility of the combined use of these drugs in this pathology.

\section{Lamins in neurology}

Biruta Kierdaszuk, Anna Łusakowska, Anna Kostera-Pruszczyk, Anna M. Kamińska

Department of Neurology, Medical University of Warsaw, Poland
Laminopathies are rare genetic disorders. The phenotype vary from muscular forms to peripheral neuropathies, lipodystrophies and premature ageing syndromes. Among cardiological symptoms conduction disturbances, systolic dysfunction of the left ventricle leading and dilated cardiomyopathy should be mentioned. The treatment for laminopathies is not known, therefore the most important are regular cardiac examinations. Cardioverter-defibrillator implantation is recommended in primary prevention of sudden cardiac death.

We present clinical, electrophysiological, neuroradiological and morphological data of 5 patients from 3 Polish families. Genetic studies of LMNA gene were performed due to cooperation with MYO-SEQ project as well as with Warsaw Genomics.

Patient number 1 (male, aged 27) was admitted due to exercise induced rhabdomyolysis at the age of 20 . Neurological examination and electromyography studies were normal. Muscle biopsy showed no abnormalities on light microscopy (LM). However, on electron microscopy (EM) primary myopathic changes with lipid droplets were observed. A variant c.356G >A in LMNA gene was identified.

Two relatives (patients number 2 and 3) from second family (females, aged 48 and 62 respectively) presented with limb-girdle muscle weakness, lack of knee reflexes and waddling gait. Electromyography studies showed myopathic pattern. There were severe myopathic changes (muscle atrophy, central nuclei, fat replacement of muscle) in muscle biopsy on LM and myofibrillar loss and abnormal mitochondria on EM. Both patients suffered from dilated cardiomyopathy, one of them had pacemaker implantation. A variant c.1130G $>$ T in $L M N A$ gene was identified.

Three sisters (patients number 3, 4 and 5, aged 57, 56 and 42 respectively) from third family were examined. All presented with limb-girdle muscle weakness, lumbar hyperlordosis and waddling gait. The age of onset varied from 20 to 30 years. Electromyography showed myopathic pattern. Muscle biopsy disclosed severe myopathic changes on LM, however, lamin A/C and desmin stainings were normal. Severe myofibrill loss was present on EM. Two patients had implantation of cardioverter defibrillators. Genetic studies confirmed variant in LMNA gene (c.1010_1016insCGAGC).

Clinical spectrum of LMNA gene mutations ranges from neurogenic muscle atrophy to myopathy. According to heterogeneous phenotype genetic studies are necessary for final diagnosis of laminopathies and its genetic counseling. 


\section{The diagnosis of the lumbar spine neuromuscular disorders in qualified athletes with use of H-reflex study}

\section{Elena Kolosova, Elena Lysenko}

National University of Physical Education and Sports in Ukraine, Scientific Research Institute, Kyiv, Ukraine

It is known that long-lasting physical exercise is accompanied by functional and structural adaptative transformations of the spinal cord structures, which are responsible for the immediate control of skeletal muscles activity. Unfortunately, inadequate exercise stress of deep dorsal spine muscles could lead to functional and structural disorders of athlete neuromuscular apparatus, such as nerve roots compression; besides the spastic limb muscles may compress underlying peripheral nerves. As a result, long-lasting nerve fibers compression leads to local demyelination and (or) axonal degeneration. One of the informative methods for quantitative analysis of the functional state of neuromuscular system could be tests with use of stimulation electromyography (EMG) in which the parameters of the $\mathrm{H}$ (Hoffmann) reflex are measured. Using $\mathrm{H}$-reflex study of soleus muscle we can recognize the state of the level $L_{v}-S_{\text {I }}$ segmental apparatus of lumbar and sacral parts of spinal cord. At our Scientific Research Institute qualified athletes specializing in biathlon, diving, rowing, canoeing, cycling etc. took part in the multimethod investigation during several years. To assess the functional state of human neuromuscular system the method of H-reflex of soleus muscle was used. Registration of EMG-signals and tibial nerve stimulation were performed using neurodiagnostic complex Nicolet Viasus Healthcare. It was found that about one third of athletes had deviations in the EMG indices from the established standard. The abnormalities were characterized by increasing of $\mathrm{H}$ - and $\mathrm{M}$-response thresholds, decrease in the amplitude of the maximal $\mathrm{H}$ - and $\mathrm{M}$-responses and amplitude ratios of maximal $\mathrm{H}$-and $\mathrm{M}$-responses, increasing of $\mathrm{H}$-and $\mathrm{M}$-responses latencies (these changes are the signs of sensory and motor nerves demyelination, respectively); changing $\mathrm{H}$-and $\mathrm{M}$-responses shape from normal two- or three-fase to polyphasic potentials (the signs of axonal degeneration of sensory and motor nerves). State of athletes, who weren't treated, got worse. But abnormal $\mathrm{H}$ - and $\mathrm{M}$-indices of athletes, who started to receive treatment at the right time, recovered to normal ones, and such athletes showed good results at the championships. Thus, H-reflex study data could be used to establish differential diagnosis of functional and structural neuromuscular system disorders, to predict the risk of injury and to monitor rehabilitation process.

\section{MELAS syndrome: clinical and morphological analysis of a unique case}

\author{
Yuliia Kuzyk, Ruslana Falion
}

Danylo Halytsky Lviv National Medical University, Lviv, Ukraine

Introduction: Mitochondrial encephalopathy, lactic acidosis and stroke (MELAS) are one of the family mitochondrial cytopathies. The purpose of the study was to analyze the unique autopsy case of MELAS syndrome.

Material and methods: We have analyzed the death of a 16-year-old boy with MELAS syndrome.

Results: It is known that the boy grew up and developed late in physical and mental development. Cerebral palsy was detected after 2.5 months. MELAS syndrome was first suspected at the age of 6 years. Genetic studies have demonstrated mutations in MT-TL1, which confirmed the diagnosis of MELAS syndrome. At the last admission to the hospital were expressed neurological symptoms - encephalopathy, spastic tetraparesis, symptomatic epilepsy with focal convulsions and tendency to secondary generalization, mental retardation of moderate degree; cardiovascular system lesions - cardiomyopathy and WPW syndrome, eye lesions - visual disk subatrophy; cerebral cachexia. Due to many organ dysfunction, the boy died on the first day of hospital stay.

A post-mortem examination revealed changes that confirmed the diagnosis of MELAS syndrome: brain lesions (cerebral hemispheres atrophy, cerebral infarctions of different time period, many focal spongiosis, petrifactions and solitary cysts of the vascular plexuses), pathology of the eye (optic disc subatrophy of both eyes), lesions of skeletal muscle (focal necrosis and muscle fiber atrophy) and cardiac muscle (hypertrophic cardiomyopathy, focal necrosis of cardiomyocytes, replacement foci and interstitial cardiosclerosis), liver lesions (fatty steatosis with onset of cirrhosis transformation) and pancreas (periductal and perivascular sclerosis). When performing morphological examination of muscle tissue by the method of modified three-chromium Gomori stain, several torn red fibers were found. Anomalous collection of mitochondria with paracrystalline inclusions, osmiophilic inclusions and mitochondrial vacuoles were detected by electron microscopy.

The cause of death on the first day of hospital stay was a shock, which could be both cardiogenic, due to the pronounced cardiac changes described above and infectious-toxic caused by bilateral purulent pneumonia.

Conclusions: This unique case demonstrates MELAS syndrome with typical clinical and morphological manifestations. The analysis of this case allows to expand the 
idea of rare syndromes and diseases in pediatric neurology, timely diagnosis of which will avoid adverse effects.

\section{Identification of the pathogenic variant in the RYR1 gene in a Ukrainian patient with severe muscle hypotonia}

\author{
Yuliia Kuzyk ${ }^{1}$, Mariya Dushar ${ }^{2}$, Olesia Kovalyk ${ }^{3}$, \\ Ruslana Falion ${ }^{1}$ \\ ${ }^{1}$ Danylo Halytsky Lviv National Medical University, Lviv, Ukraine \\ ${ }^{2}$ Institute of Hereditary Pathology NAMS, Lviv, Ukraine \\ ${ }^{3} \mathrm{CI}$ LOR “Lviv Regional Pathology Bureau”, Lviv, Ukraine
}

Introduction: Central core disease (CCD) is a dominantly inherited congenital myopathy allelic to malignant hyperthermia $(\mathrm{MH})$ caused by mutations in the RYR1 gene on chromosome 19q13.1.

Material and methods: The case of central core disease. The NGS analysis of RYR1 gene and Sanger sequencing next conformation was performed after birth.

Results: The boy was referred for clinical genetic diagnostics at the age of 6 months. He was born at term after an uneventful pregnancy; the parents were healthy and unrelated and birth weight was $3370 \mathrm{~g}$, length $53 \mathrm{~cm}, 6 / 7$ Apgar score, the baby had respiratory disorders. He spent 7 days in the intensive care unit. After birth, echocardiography was performed and heart defect - ventricular septal defect $2 \mathrm{~mm}$. Brain MRI revealed hydrocephalus, a variant of Dandy Walker's. A dysmorphological examination showed - hypomic face, pectus excavatum, cryptorchidism, severe muscle hypotonia. The genetic testing revealed pathogenic variant c. 2505 del p.Pro836Leufs ${ }^{\star} 48$, it was identified in RYR1. The sequencing of RYR1 gene of both parents did not detect mutations. The RYR1 gene is associated with autosomal dominant and recessive central core disease, autosomal recessive congenital myopathy with fiber-type disproportion, and autosomal recessive multiminicore disease. It is also associated with autosomal dominant centronuclear myopathy and malignant hyperthermia susceptibility type 1 . Malignant hyperthermia is a pharmacogenetic disorder. It is not a disease per se but rather a genetic predisposition to develop a serious adverse reaction in response to exposure to certain potent inhalational anaesthetics and depolarising muscle relaxants.

Muscle biopsy was performed in this hematoxylin and eosin section, much of the muscle consists of tiny fibers intermixed with an occasional larger myofiber. Adipose replacement has formed. Because of the extensive muscle atrophy, the intramuscular nerve twigs appear quite prominent. At higher magnification in the Masson trichrome stain section, a few near-normal myofibers are intermixed with many small and tiny fibers. Endomysial connective tissue was greatly increased.

Thus, the patient, according to genetic analysis and biopsy data, has a central core disease associated with malignant hyperthermia.

Conclusions: The diagnosis should be considered in any child or young adult presenting with weakness, hypotonia in infancy, and/or skeletal abnormalities.

\section{Focal cytoarchitectural cortical malformations with genetic background}

\section{Milena Laure-Kamionowska ${ }^{1}$, Paweł Gawliński ${ }^{2}$ Izabela Plaskota², Wojciech Wiszniewski ${ }^{2,3}$}

${ }^{1}$ Department of Experimental and Clinical Neuropathology, Mossakowski Medical Research Centre, Polish Academy of Sciences, Warsaw, Poland

${ }^{2}$ Department of Medical Genetics, Institute of Mother and Child, Warsaw, Poland

${ }^{3}$ Department of Molecular and Medical Genetics, Oregon Health and Science, University, Portland, USA

We present various patterns of focal cytoarchitectural cortical malformations in fetal and neonatal brains with genetic disorders. Genomic analysis revealed mutations in SMPD4, ETFDH genes, Pallister-Killian syndrome, trisomy chromosome 13 and 18. The neuropathological examination showed the wide spectrum of disorganized cerebral cortical architecture.

The subtle malformations were mostly restricted to external cortical layers. The fusion of the molecular layers and the absence of normal tertiary sulci were most frequently observed. The abnormalities of the glial-pial with cells transgressing into the meninges and forming there heterotopias were found. In the molecular layer nests of ectopic neurons were arranged in clusters which formed dense groups on the border of pial surface. The multiple overmigrated neurons and glial cells in the first molecular layer disordered its normal structure. Cells were either loosely dispersed or arranged in multiple waves.

An abnormal organization of all layers of cortical mantle was less common. Foci with irregularity of laminar or radial structure of the cortex were observed.

The abnormal glial-pial barrier, improper placement of radial glia, incorrect location of Cajal-Retzius cells and disturbed their interaction with other cortical components led to overmigration of neurons. Overmigrated neurons lost their destination, failed to move to deeper layers of developing cortex and settled in the molecular zone. This condition resulted in various cytoarchitectural cortical malfor- 
mations ranging from subtle neuronal ectopias in marginal layer to complete disorganization of cortical mantle.

\section{The spectrum of neuropathological changes in case of intractable epileptic encephalopathy}

\section{Milena Laure-Kamionowska ${ }^{1}$, Krystyna Szymańska ${ }^{1}$, Krzysztof Szczałuba², Mariusz Furmanek ${ }^{3}$, Rafał Płoski}

1Department of Experimental and Clinical Neuropathology, Mossakowski Medical Research Centre, Polish Academy of Sciences, Warsaw, Poland

${ }^{2}$ Department of Medical Genetics, Medical University of Warsaw, Poland

${ }^{3}$ Department of Diagnostic Imaging, Centre of Postgraduate Medical Education, Warsaw, Poland

Intractable epileptic encephalopathy is the prominent feature of Rigidity and multifocal seizure syndrome, lethal neonatal (RMFSL) referred/related to homozygous pathogenic variants in the BRAT1 gene. The syndrome is characterized by the early onset, severe course, microcephaly, rigidity, multifocal seizures resistant to treatment with myoclonic jerks and early death. We present siblings which had polymorphic seizures resistant to treatment and significantly increased muscle tension since their first days of life. Girl died at age of 6 months, brother at age of 12 months.

Genetic examination (NGS sequencing) revealed in patient 2 the presence of homozygous variant in BRAT1 gene located in exon 9.

Neuropathological examination of hypoplastic brain of patient 2 revealed a wide range of changes. The main feature was the cerebellar destruction with generalized damage of granular neurons in the internal layer and eosinophilic spheroids in the molecular layer. In the cerebral hemispheres disseminated cortical lesions within layers II-V and degeneration with microvacuolization in the middle layers were stated. The generalized retardation of myelination of cerebral tracts was observed. The pattern of progression of myelination was normal; an overall delay of myelination was about several months. It is difficult to determine what morphological changes can be attributed to the cause of epilepsy and what are its results or consequences. Lobular atrophy of the cerebellum, diffuse cortical atrophy, lesions of the Ammon's horn and subpial gliosis can be observed in long-lasting epilepsy. However it appears that the disruption of the myelination process, as well as neurodegenerative changes of the cerebellar granule cells may be associated with the gene's function.

\section{Transcriptional profiling of pediatric brain tumors}

\section{Maria Łastowska}

Department of Experimental and Clinical Neuropathology, Mossakowski Medical Research Centre, Polish Academy of Sciences, Warsaw, Poland

High grade pediatric brain tumors are characterized by a continuing high mortality rate, especially tumors of embryonic origin. So far, usually clinical factors classify patients into the prognostic groups, but this stratification is still not adequate for a number of children. For example, in ependymoma discrepancies exist in finding a definitive correlation between clinical outcome and such factors as histologic grade, location or treatment modality.

Microarrays technologies for whole genome analysis at the gene expression levels were applied to several types of childhood brain tumors. The results of transcriptional profiling revealed molecular heterogeneity of all analyzed tumors, which included medulloblastoma, ependymoma, high grade gliomas, primitive neuroectodermal tumors (PNETs) and rhabdoid tumors (AT/RTs). Importantly, identification of the transcriptional subgroups is clinically relevant. For example, patients with medulloblastoma WNT group have a favorable outcome and a reduction of the radiation dose for those patients should be considered, thus potentially reducing the severe side effects of the current treatment.

Nevertheless, microarrays analysis is a challenging approach for routine diagnostic purposes. Pathologists, as a rule, store tumor biopsies as formalin-fixed parafine-embeded (FFPE) material in order to preserve cellular and structural integrity of the original tumor specimen. Only some hospitals store tumor material as a frozen tissue allowing for subsequent molecular studies.

For analysis of gene expression level in tumor material the Nanostring nCounter Technology may be applied because of its compatibility with FFPE specimens. The assay provides a method for direct detection of mRNAs with molecular barcodes without the use of amplification or reverse transcription, which steps are essential in analysis of microarrays or Real-Time PCR. In consequence, it is possible to analyze degraded RNA for reliable assessment of genes expression levels.

Therefore, the Nanostring nCounter Technology may be applied for transcriptional subgrouping of high grade pediatric brain tumors. This method has already been applied formolecularclassification of medulloblasomausing 22 marker genes, and recently for molecular classification of infratentorial ependymomas. The later tumors include two molecularly distinct subgroups, ependymoma A 
(EPN-A) and ependymoma B (EPN-B), which differ in terms of survival rate for patients. Analysis of infratentorial ependymomas using Nanostring method and 10 marker genes identifies patients with favorable prognosis group $B$, which shows high expression of EPN-B signature genes: NELL2, CEP83, NXNL2, DNAH1 and C9orf72 as opposite to low expression of EPN-A signature genes: LAMA2, CXorf67, IGSF, ALDH1L1 and SLC6A13.

In summary, gene expression RNA profiling from FFPE tumor samples using NanoString nCounter system may be a useful approach for detection of tumors which show favorable clinical behaviour.

Funded by the National Science Centre, Poland (2016/21/B/NZ2/01785).

\section{The frequency of BDNF Val66Met (rs6265) polymorphism in Polish population - preliminary studies}

\section{Paulina Małczyńska, Wiktoria Radziwonik, Filip Tomczuk, Ewelina Elert-Dobkowska, Anna Sułek}

Laboratory of Molecular Basis of Neurodegenerative Diseases, Department of Genetics, Institute of Psychiatry and Neurology, Warsaw, Poland

Brain derived neurotrophic factor (BDNF) is one of the most important neurotrophic factors responsible for the synaptic neuroplasticity, neuronal differentiation and neuronal survival. A single nucleotide polymorphism (SNP) Val66Met (rs6265) might play very important role in the BDNF level. Previous research shows that people with Met in 66 position of BDNF mRNA have decreased BDNF level in serum. The presence of the Val66Met polymorphism might be associated with the higher risk of psychiatric disorders (depression, schizophrenia) or neurodegenerative diseases (Parkinson's disease). The aim of this study was to investigate the frequency of Val66Met polymorphism in the Polish population.

200 healthy subjects without any neurological or psychiatric disorders were screened for the presence of Val66 Met polymorphism. The restriction enzyme Eco72I (PmII) which recognizes the CAC-GTG sequence was used for the determination of BDNF polymorphism. For the confirmation of proper PCR RFLP analysis, chosen subjects were analyzed by Sanger sequencing. Pearson's chi-squared test was used to test deviation from the Hardy-Weinberg principle.

PCR RFLP analysis shows that 147 individuals do not have a BDNF Val66Met polymorphism, 48 are heterozygotes and 5 cases are recessive homozygotes carrying SNP on two allels. Pearson's chi-squared test shows that frequency for
G allel is $p=0.8550$ and $\mathrm{A}$ allel is $q=0.1450$. Chi-square $=$ $0.2056, \mathrm{df}=1$.

Obtained results shows that BDNF polymorphism Val66 Met might be detect in $26.5 \%$ of Polish population and that is in Hardy-Weinberg equilibrium.

\section{The incorporation of genetic markers in the routine diagnostic approach of diffuse gliomas}

\section{Ewa Matyja}

Department of Experimental and Clinical Neuropathology, Mossakowski Medical Research Centre, Polish Academy of Sciences, Warsaw, Poland

Advances in the molecular pathology of brain tumours have provided the basis for an integrated diagnostic approach that forces the incorporation of new molecular markers into routine diagnostic practice. However high cellular and genomic heterogeneity of gliomas makes diagnosis difficult and limits therapeutic options. Nevertheless, it appears that many genomic markers including IDH1/2 mutations, MGMT gene promoter methylation, and TERT promoter mutation play an important role as diagnostic and/or prognostic agents. According to the WHO 2016 classification, five main molecular subgroups of diffuse gliomas can be distinguished based on three molecular biomarkers: mutation of $I D H, 1 p / 19 q$ codeletion, and histone $\mathrm{H} 3.3$ mutation. Additional molecular changes, including P53, ATRX and TERT promoter mutation are also useful in the differential diagnosis. Immunohistochemical markers provide insight into the underlying molecular changes and are helpful in the identification of diffuse astroglial and oligodendroglial tumours. It is postulated that combination of tumor morphology, immunophenotype for IDH1-R132H, ATRX and p53, accompanied by $1 p / 19 q$ status is most suitable to classify the majority of diffuse gliomas. Generally, morphology of diffuse astrocytoma, loss of ATRX or strong p53 staining are sufficient to diagnose an astrocytoma of the appropriate grade without $1 p / 19 q$ assessment. In the other hand, $1 p / 19 q$ codeletion is associated with the TERTp mutation and commonly excludes the ATRX mutation, thus anti-ATRX immunohistochemistry has been proposed as a surrogate of $1 p / 19 q$ codeletion marker concerning IDH-mutant gliomas. However, some gliomas with $1 \mathrm{p} / 19 \mathrm{q}$ codeletion show a loss of ATRX nuclear expression, as well as not all IDH-mutant gliomas with ATRX immunostaining are 1p/19q codeleted.

Although the development of molecular research allows us to better understand the biology of brain tumors, 
there are still questions about current classification criteria i.e. whether a heterogeneous group of diffuse astrocytomas IDH-wildtype, classified as grade II or III according to WHO, should be considered as a separate entity, although a certain percentage of them may clinically behave like GBM IDH-wildtype. It has been proposed that these astrocytomas, although they do not fulfill histological criteria of GBM, may be classified as "Diffuse astrocytic glioma, wild type IDH, with molecular features of glioma, WHO grade IV". For cases in which full molecular information is not available or tests cannot be performed, the diagnosis "not otherwise specified" (NOS) may be used, while cases where the obtained molecular information does not fit into the existing diagnostic category can be termed "not elsewhere classified" (NEC).

In the current classification scheme, despite the discrepancy between histology and molecular classification, molecular markers appear to be a more significant prognostic factor than histological features.

\section{Hypomyelinating leukodystrophies: diagnostic difficulties of particular entities}

\section{Hanna Mierzewska}

Department of Child and Adolescent Neurology, Institute of Mother and Child, Warsaw, Poland

Leukodystrophies hypomyelinating (HLD) are genetically determined ultra-rare diseases of the white matter of the brain characterized by permanent lack or shortage of myelin. To date (2019), in the McCusick's catalog about 18 disease entities have been included. However, the number is higher because the inclusion criteria to given subgroup depends on the dominant symptoms that prevail in particular disease, e.g. convulsiones, bone changes, brainstem atrophy etc. which may result in classification into another sub-group. Additionally to this day, they are still HLD with an undetermined ground. In infancy hypomyelination should be differentiated from delayed myelination. The etiopathogenesis of HLD is complex: it may be due to deficiencies in the components of myelin, membrane or cytoplasmic proteins, and of products of housekeeping genes. HLD are most frequently diagnosed in children and adolescents, but they can also be detected in adults. They have a different course and MRI picture than more frequent demyelinating leukodystrophies and are often misdiagnosed as cerebral palsy. The MRI of HLD is characterized by a hyperintensive signal in the $T 2$ and FLAIR and hypo-/isointensive in T1 sequences. Some of HLD have additional symptoms that may be an excellent "key to diagnosis" such as the involvement of the basal ganglia, cerebellum, cataracts, hypodontia or hypogonadism. Diagnosis is based on DNA analysis.

Several molecularly diagnosed units will be presented.

\section{Hidden carriership of spinal muscular atrophy - implication for genetic canceling}

\section{Katarzyna Ozdarska, Agata Raniszewska, Marta Beć, Janusz G. Zimowski}

Department of Genetics, Institute of Psychiatry and Neurology, Warsaw, Poland

Spinal muscular dystrophy (SMA) is a genetically determined neurodegenerative disease that, as a result of the loss of the motor neurons in the spinal cord, leads to muscle denervation, and then to their progressive weakness and atrophy.

The disease is inherited in an autosomal recessive manner, caused by both parallelic mutations of the SMN1 gene located on chromosome 5 . In $96 \%$ of patients, exon 7 homodeletion of the SMN1 gene is detected, while the remaining deletions within one allele are accompanied by a point mutation in the other.

SMA carrier tests are based on determining the number of copies of the SMN1 gene - detection of one copy of this gene is interpreted as an unambiguous finding of a carrier status, detection of two or more copies of the SMN1 gene is interpreted as an exclusion of SMA carrier. Unfortunately, the frequent phenomenon of SMN1 and SMN2 gene conversion (difficult to identify directly) changes their number within the same chromosome. In family studies of inheritance of SMA carrier among 97 families, we detected 7 cases of hidden carrier consist in the presence of two copies of the SMN1 gene on one chromosome 5 with the simultaneous lack of this gene on the other chromosome 5. In addition, we detected 3 cases that are either cases of hidden carrier or newly formed mutations.

It is known that in Poland at least one in 40 people has a mutation in its genome responsible for spinal muscular atrophy. The result of a carrier test in which it is excluded (based on the presence of two copies of the SMN1 gene) requires special care. The resolution can be brought by in-depth pedigree analysis and molecular research. 


\section{Expression of genes involved in amyloidogenic processing of the amyloid protein precursor in the CA1 area of the hippocampus after brain ischemia}

\author{
Ryszard Pluta ${ }^{1}$, Marzena Ułamek-Kozioł ${ }^{1,2}$, \\ Stawomir Januszewski ${ }^{1}$, Stanisław J. Czuczwar ${ }^{3}$ \\ ${ }^{1}$ Laboratory of Ischemic and Neurodegenerative Brain Research, \\ Mossakowski Medical Research Centre, Polish Academy \\ of Sciences, Warsaw, Poland \\ ${ }^{2}$ First Department of Neurology, Institute of Psychiatry and \\ Neurology, Warsaw, Poland \\ ${ }^{3}$ Department of Pathophysiology, Medical University of Lublin, \\ Lublin, Poland
}

It is believed that brain ischemia can be causally related to Alzheimer's disease. Changes in the expression of genes for both $\beta$ - and $\gamma$-secretases and amyloid protein precursor in the CA1 area of the hippocampus after 10-minute brain ischemia in rats surviving 2, 7 and 30 days were assessed by quantitative reverse-transcriptase PCR assay. In the CA1 region of the hippocampus, the maximal significant overexpression of the $\beta$-secretase gene was recorded on the second day after ischemia, while on day 7 the expression of this gene was only slightly increased. But on day 30 , the expression of this gene was only moderately reduced from the control values. The presenilin 1 and 2 genes showed the same trend of expression as the $\beta$-secretase gene. The amyloid protein precursor gene had downregulated expression on day 2 after ischemia, but on days 7-30, a considerable reverse tendency occurred. Thus, the presented study indicate that the induction of $\beta$-secretase, presenilin 1 and 2, and amyloid protein precursor genes is associated with the response of neurons in the CA1 area of the hippocampus to transient brain ischemia. These observations suggest that ischemic episode is a triggering amyloidogenic process factor extremely dangerous for the brain. The study will help to understand progressive damage after brain ischemia, and both delayed accumulation of amyloid and Alzheimer's disease-type neuropathology development. In addition, the present data demonstrate that ischemic brain injury activates pyramidal neuronal death in the CA1 area of the hippocampus in a manner dependent on the $\beta$-amyloid peptide, thus defining a new and important mechanism for regulating the survival and/ or death of post-ischemic neurons.

\section{$A P O E$ cluster polymorphisms and risk of Alzheimer's disease}

\author{
Michal Prendecki ${ }^{1}$, Marta Kowalska ${ }^{1}$, \\ Jolanta Florczak-Wyspianska², Jan Ilkowski³, \\ Wojciech Kozubski ${ }^{2}$, Jolanta Dorszewska ${ }^{1}$
}

\author{
${ }^{1}$ Laboratory of Neurobiology, Department of Neurology, Poznan \\ University of Medical Sciences, Poznan, Poland \\ ${ }^{2}$ Chair and Department of Neurology, Poznan University \\ of Medical Sciences, Poznan, Poland \\ ${ }^{3}$ Department of Emergency Medicine, Poznan University \\ of Medical Sciences, Poznan, Poland
}

Introduction: Alzheimer's disease (AD) is a most prominent progressive cognitive disorder in older adults, characterized by excessive brain deposition of amyloid $\beta$ $(\mathrm{A} \beta)$ and neurofibrillary tangles (NFT). The most common, sporadic form of the disease is in $70 \%$ associated with genetic risk factors, e.g. in $A P O E$ locus. The studies on effects of APOE neighboring genes: TOMM4O and APOC1 are neglected in $A D$ patients from Polish population.

Aim of the study: To analyze the frequency of chosen polymorphisms in the APOE cluster, in TOMM40 gene (rs10524523 and rs2075650), in APOE gene (rs429358 and rs7412) and in APOC1 locus (rs4420638) in Polish AD patients in respect to age of onset.

Material and methods: To the study were recruited 230 individuals above 60 years of age: 88 AD patients, 80 control volunteers without (UC) and 62 control persons with $(R C)$ positive family history of AD.

The $A P O E$ genotyping was performed by mismatch-primer qPCR, TOMM40 genotypes were determined by HRM and capillary electrophoresis, and APOC1 was analyzed by HRM.

Results: It has been confirmed that APOE E4 was significant risk factor of developing $A D$ and remained in linkage disequilibrium with TOMM40'523-L allele. Similarly, both TOMM40'650-G and APOC1'638-G alleles were overrepresented in AD group and were significant risk factors of developing dementia. Moreover, the impact of both variants remained significant in APOE E4 non-carriers. The co-occurrence of three polymorphisms TOMM40'523-L, '650-G and APOC1'638-G was associated with 6x greater risk of developing dementia. The pathogenic polymorphisms were also more common in comparative group (RC vs. UC). Subsequently, the presence of analyzed polymorphisms was associated with earlier manifestation of $\mathrm{AD}$, especially in homozygous carriers: TOMM40'523-S/S and -L/L (vs. -VL/VL), as well as in TOMM40'650-G/G and APOC1'638-G/G carriers (vs. A/A genotypes). Finally, in the AD patients with protective haplotype TOMM40'523TOMM40'650-APOC1'638 - VL-A-A, the disease devel- 
oped 14 years later than in patients with less favorable arrangement: L-G-G.

Conclusions: It seems that polymorphisms rs10524523 and rs2075650 in TOMM40 gene, rs429358 and rs7412 in APOE gene and rs4420638 in APOC1 locus contribute to the risk of developing $A D$, and are significantly associated with age of dementia onset.

\section{The application of hyperbaric oxygen as an addition to anti-tumour treatment with investigational compounds against glioblastoma cells in vitro}

\section{Emanuela Pucko, Robert P Ostrowski, Maria Zielińska, Ewa Matyja}

Department of Experimental and Clinical Neuropathology, Mossakowski Medical Research Centre Polish Academy of Sciences, Warsaw, Poland

The hyperbaric oxygen ( $\mathrm{HBO}$ ) has been used as an adjuvant therapy for glioblastoma. It deserves study whether $\mathrm{HBO}$ can be used in combination with different antitumor compounds, including inhibitors of CK2 kinase, to potentiate therapeutic effect. The objectives of this study were to demonstrate the feasibility of $\mathrm{HBO} / \mathrm{CK} 2$ kinase inhibitor therapy in vitro and investigate the effect of combined therapy on the viability of malignant glioma cells.

HBO of cultured cells was performed with the use of small cylindrical hyperbaric chamber equipped with shelves. After gassing at the atmospheric pressure, the compression started at a rate of $0.1 \mathrm{bar} / \mathrm{min}$ and upon reaching the desired depth of 2 atmospheres absolutes (ATA) the hyperbaric session begun. At 60 min decompression was started at a rate $0.1 \mathrm{bar} / \mathrm{min}$. The changes in the gas pressure level were regulated by operating the main valve at the top of the gas tank with certified gas mix.

T98G cells were cultured in MEM medium supplemented with CK2 inhibitor at different concentrations. The cultures treated with the inhibitor were assigned to normoxic conditions for 24 hours or 1 hour of hyperbaric oxygen ( $\mathrm{HBO}, 97.5 \% \mathrm{O}_{2} / 2.5 \% \mathrm{CO}_{2}$, pressure of $\left.2 \mathrm{ATA}\right)$ followed by 23 hours of normoxia $\left(21 \% \mathrm{O}_{2} / 5 \% \mathrm{CO}_{2} / 78 \%\right.$ $\mathrm{N} 2$ ). The viability of glioma cell lines was determined at 24 hours post-treatment by CellTiter $96{ }^{\circledR}$ AQueous One Solution Cell Proliferation Assay.

The study showed that the administration of HBO even alone caused a reduction in the viability of glioblas- toma cells, whereas CK2 inhibitor reduced the viability of cells at higher levels within the applied concentration range. HBO significantly enhanced the cytotoxic effect of CK2 inhibitor upon combined treatment. Noteworthy, under the influence of hyperbaric oxygen, CK2 inhibitor could reduce the viability of tumour cells already at a low concentration after 24 hours.

$\mathrm{HBO}$ can be safely used as an adjuvant treatment in the combination therapy with CK2 inhibitors, increasing their efficacy. The method is straightforward, feasible and may allow to easily obtain the samples for cancer cell pathology, molecular biology and epigenetic studies.

Acknowledgement: The research was supported by the Foundation for the Development of Diagnostic and Therapy, Warsaw.

\section{The development and application of NGS panels in diagnostics of the hereditary neurodegenerative disorders}

\section{Wiktoria Radziwonik ${ }^{1}$, Ewelina Elert-Dobkowska ${ }^{1}$, Iwona Stępniak², Karolina Ziora-Jakutowicz ${ }^{2}$, Filip Tomczuk $^{1}$, Paulina Małczyńska ${ }^{1}$, Anna Sułek ${ }^{1}$}

${ }^{1}$ Laboratory of Molecular Basis of Neurodegenerative Diseases, The Institute of Psychiatry and Neurology, Warsaw, Poland ${ }^{2}$ Genetic Counselling Unit, Department of Genetics, The Institute of Psychiatry and Neurology, Warsaw, Poland

The diagnostics for rare neurodegenerative disorders are challenging due to their genetic and clinical heterogeneity. Therefore, there is a need for implementation a time- and cost-effective technology - Next Generation Sequencing (NGS). The aim of our study was to develop the targeted gene NGS panels based on many years of our genetic and clinical experience. Panel I contains probes for coding sequences for 152 known genes associated with spinocerebellar ataxias and hereditary spastic paraplegias; panel II - 118 genes related with amyotrophic lateral sclerosis and frontotemporal dementias, HD-like phenotype, Parkinson's disease, Alzheimer's disease, dementias, dystonias, leukodystrophies; panel III - 89 genes causing muscular dystrophies, myasthenias, myotonias, myopathies.

The list of implemented genes were based on data in GeneReviews, scientific reports and literature. DNA was isolated from blood by using MagNA Pure Compact Nucleic Acid Isolation Kit according to the manufacturer's procedure.

The workflow of NGS involves several steps: (1) Bead Cleanup to remove EDTA from input genomic DNA; (2) library preparation with (a) Enzymatic Fragmentation, (b) 
End Repair \& A-tailing, (c) Adapter Ligation; (3) library amplification; (4) pooling the amplified, captured library; (5) sample hybridization with SeqCap EZ Probe Pool (the complete set of biotinylated long oligonucleotide probes provided by Roche company); (6) washing and recovering captured DNA after hybridization by using SeqCap Pure Capture Bead Kit; (7) amplification of captured DNA using LM-PCR and Cleanup; (8) sequencing on MiSeq instrument.

The analysis of genetic variants were performed by using BaseSpace Variant Interpreter, Mutation Taster, ClinVar, HGMD, LOVD, OMIM and interpretation were according to the ACMG Standard and Guidelines. The presence of all detected pathogenic/likely pathogenic variants were confirmed by using the Sanger sequencing on genetic analyzer ABI 3130.

In total, 64 patients were examined, in 37 (57.8\%) a pathogenic/likely pathogenic mutations were detected.

To confirm the pathogenicity of unknown mutations, it is necessary to study at least a family segregation of detected variant.

\section{Tailor - made molecular genetic test in gliomas}

Agata Ryfa ${ }^{1}$, Anna Majdańska ${ }^{1}$, Hanna Morzyńska ${ }^{1}$, Tadeusz Szylberg ${ }^{1}$, Jacek Furtak ${ }^{2}$, Krystyna Soszyńska ${ }^{1}$

${ }^{1}$ Department of Pathology, Clinical Genetics and Molecular Pathology Laboratory, $10^{\text {th }}$ Military Research Hospital and Polyclinic, Bydgoszcz, Poland

${ }^{2}$ Department of Neurosurgery, $10^{\text {th }}$ Military Research Hospital and Polyclinic, Bydgoszcz, Poland

Insights into the molecular biomarkers of central nervous system tumors have radically changed the tumor diagnosis and classification. They have become an excellent tools and has shifted the diagnosis from the morphology of a tumor to an integrated based on morphologic and molecular features.

The integrated diagnosis is beneficial for patients with glioma have implications for diagnosing, prognosis and selecting treatment. Test of methylation status of MGMT gene implies whether the patient can benefit from treatment with temozolomide simultaneously with radiotherapy (observed increased lifetime). Presence of $1 p / 19 q$ co-deletion has significant prognosis relevance for oligodendrogliomas. IDH1/IDH2 mutations are diagnostic and prognostic factors.

Stereotactic biopsy has became a diagnostic tool to obtain tissue samples to diagnosis patients with brain tumors where resection is not considered. The size of the sample obtained with this technique is very limited, and usu- ally only histopathological diagnosis is evaluated. In order to improve a diagnostic process for glioma small size samples acquired by stereotactic brain biopsy, the molecular genetics analyses were introduced in our laboratory.

Tumor tissues were collected during surgery and from stereotactic biopsy from patients of Department of Neurosurgery, $10^{\text {th }}$ Military Hospital in Bydgoszcz.

The materials tissue samples were formalin-fixed, paraffin-embedded (FFPE) then diagnosed by pathomorpholog and graded according to the WHO Classification of tumors of the Central Nervous System 2016.

The MLPA method (Multiplex Ligation-dependent Probe Amplification) was used to detect co-deletion $1 \mathrm{p} / 19 \mathrm{q}$ and IDH1(R132H,R132C)/IDH2(R172K,R172M) mutations and to check MGMT gene promoter methylation.

The fact we have developed and validated procedure for determination of mutations IDH1/IDH2, co-deletion $1 p / 19 q$ and methylation status of MGMT gene, using small biopsy specimens from patients with glioma.

Molecular genetic analysis can be successfully applied to stereotactic biopsies of small size samples helping in correct tumor classification, its expansion prognosis and choosing optimal, customized treatment.

\section{Influence of polymorphisms in APOE, APOC1, and $A C E$ genes on the risk of Alzheimer's disease}

\section{Marzena Skrzypa ${ }^{1^{*}}$, Natalia Potocka ${ }^{1^{*}}$, Halina Bartosik-Psujek ${ }^{2,3}$, Marcin Wiącek ${ }^{2,3}$, Marcin Braun ${ }^{4}$, Izabela Zawlik ${ }^{1,2}$}

\author{
${ }^{1}$ Laboratory of Molecular Biology, Centre for Innovative \\ Research in Medical and Natural Sciences, Faculty \\ of Medicine, University of Rzeszow, Poland \\ ${ }^{2}$ Institution of Experimental and Clinical Medicine, Faculty \\ of Medicine, University of Rzeszow, Poland \\ ${ }^{3}$ Clinical Department of Neurology Rzeszow State Hospital \\ No. 2, Rzeszow, Poland \\ ${ }^{4}$ Department of Pathology, Chair of Oncology, Medical \\ University of Lodz, Poland \\ *Equal contribution
}

Alzheimer's disease (AD) is one of the most common neurodegenerative diseases, which is a serious health problem for societies that live longer. Polymorphisms of selected genes may play an important role in development of $\mathrm{AD}$. Mild cognitive impairment $(\mathrm{MCl})$ is considered an intermediate state between normal aging and dementia, mainly Alzheimer's type. The aim of this study was to assess the influence of polymorphisms of APOE, APOC1, and $A C E$ genes on the risk of $A D$. We examined the rela- 
tionship between these gene polymorphisms (separately and in the following combinations: $A P O E$ and $A P O C 1$, $A P O E$ and $A C E$ ) and susceptibility to $A D$ and $M C l$.

The study included 63 patients with $A D$ (mean age = 74.88), 35 patients with $\mathrm{MCl}$ (mean age $=66.45$ ) and 35 healthy subjects (mean age $=66.05$ ). DNA was isolated from WBC (White blood cells). Polymorphisms in APOE, APOC1 and $A C E$ genes was evaluated by using PCR-RFLP and PCR.

We observed a very strong correlation between the presence of $A P O E \& 4$ and $A P O C 1$ II (insertion/insertion) and ID (insertion/deletion) genotypes and the risk of $\mathrm{MCl}$ and $\mathrm{AD}$ development. We also observed that people with DD (deletion/deletion) genotype in the $A C E$ gene were diagnosed $\mathrm{MCl}$ at a later age. This results suggest the $A P O C 1$ insertion allele, in combination with $A P O E \& 4$, may be a potential risk factor for developing AD. Moreover, the ACE polymorphism may influence on $\mathrm{MCl}$ age of onset.

\section{Transcriptional profiling and histopathological analysis of pediatric infratentorial ependymoma tumours}

Anna Antonina Sobocińska ${ }^{1}$, Magdalena Niemira², Anna Szałkowska², Bartosz Wojtaś ${ }^{3}$, Joanna Trubicka ${ }^{4}$, Wiesława Grajkowska ${ }^{4}$, Ewa Matyja ${ }^{1}$, Maria Łastowska ${ }^{1,4}$

${ }^{1}$ Department of Experimental and Clinical Neuropathology, Mossakowski Medical Research Centre, Polish Academy of Sciences, Warsaw, Poland

${ }^{2}$ Clinical Research Centre, Medical University of Bialystok, Poland

${ }^{3}$ Neurobiology Center, Nencki Institute of Experimental Biology, Warsaw, Poland

${ }^{4}$ Department of Pathology, The Children's Memorial Health Institute, Warsaw, Poland

Introduction: Pediatric ependymomas are molecularly heterogeneous group of tumours that arise from ependymal lining of the ventricles and the central canal. Most ependymomas are localized intracranially, half of them in the infratentorial region. Infratentorial cases are diagnosed mostly in patients of younger age and recent studies show that these tumours consist of two molecular subgroup: A (EPN-A) and B (EPN-B). These subgroups differ in biological behavior and therefore it is crucial to establish new molecular markers that will help diagnosis process and stratification of patients into risk groups. DNA methylation-based approach is recommended for molecular classification of ependymoma, however, this test cannot be performed on formalin-fixed, paraffin-embedded (FFPE) material.
Material and methods: In this study we investigated the expression level of marker genes at the RNA level from FFPE tumour samples using NanoString nCounter system analysis. For detection of ependymal subgroup a custom NanoString CodeSet was applied which consisted of 10 marker genes: LAMA2, CXorf67, IGSF, ALDH1L1, SLC6A13 for a subgroup EPN-A and NELL2, CEP83, NXNL2, DNAH1, C9orf72 representing EPN-B subgroup. Tumours were analyzed according to NanoString Technologies procedures for hybridization, detection and scanning. Raw counts for each gene underwent technical and biological normalization using nSolver4.0 software. Histopathological analysis of the examined cases was made to determine if there are any morphological differences between EPN-A and EPN-B.

Results: After performing HeatMap analysis of 30 cases of ependymomas, 5 cases showing high expression of EPN-B signature genes were identified. Clinical data showed that patients with EPN-B were statistically older than patients with EPN-A. Moreover, none of EPN-B patients exhibited a relapse and all are still alive. The clinical characteristics of the analyzed cases are consistent with previous findings for these specific molecular subgroups.

All tumours classified as ependymomas Grade II and III according to WHO showed heterogeneity of the histopathological picture, however in our series there were no significant differences between EPN-A and EPN-B subgroup. The specific histopathological features of ependymomas in the form of less or more pronounced perivascular pseudo-rosettes were seen in almost all cases. True ependymal rosettes were observed only in one case. In some cases the delicate, branched capillaries and clear cell appearance resembling oligodendroglioma could be noticed. The nodules of high cell density within regions of low cell density background were occasionally seen. Anaplastic EPNs exhibited densely cellular lesions, composed of uniform, closely packed accompanied by microvascular proliferation, necrosis and mitotic figures.

Conclusions: The expression of RNA profiling from FFPE tumour samples using NanoString nCounter System maybe an useful approach for detection of EPN-B tumors that show favorable clinical behavior. No significant histopathology differences between EPN-A and EPN-B were found in our series.

\section{Virtual reality in clinical neuroscience}

\author{
Ewa Sokołowska ${ }^{1}$, Beata Sokołowska ${ }^{2}$ \\ ${ }^{1}$ Department of Clinical Psychology, Faculty of Social Sciences, \\ John Paul II Catholic University of Lublin, Poland \\ ${ }^{2}$ Bioinformatics Laboratory, Mossakowski Medical Research \\ Centre Polish Academy of Sciences, Warsaw, Poland
}


As we have observed, the $21^{\text {st }}$ century is a new important and interesting era for medical information (mobile) technologies in clinical medicine and is described by the term "e-Health \& m-Health" (http://www.euro.who.int/ en/ehealth). E-Health is a young field of medicine, defined by Eysenbach (J Med Internet Res 2001; 3(2): e20) as "the intersection of medical informatics, public health and business, referring to health services and information delivered or enhanced through the Internet and related technologies". People with multimorbidity, especially elderly people or patients with fatal/rare genetic diseases, have complex health and social needs, and require an integrated care approach. In this respect, e-Health \& m-Health could be supportive. In the medical area, therapeutic Virtual Reality (VR) is an innovative treatment modality to manage a wide range of health conditions and is gaining considerable attention (JMIR Ment Health 2019; 6(1): 1-14). VR and serious games technologies have been used to evaluate and treat a wide variety of medical education and modelling, surgery, psychiatry, psychotherapy, cyberpsychology, and cognitive or motor conditions including physical therapy/ motor rehabilitation (Folia Neuropathol 2018; 56(3): 270271; Cyberpsychol Behav Soc Netw 2019; 22(1): 82-96; IJIRCCE 2019; 7(7): 3555-3562). Recently, the phrase "intelligent health" is sometimes used to indicate the transformation/analysis of electronic (medical records) databases, obtained by means of e-Health tools, into knowledge and the consequent integration of real-time self-monitoring with assessment of the patient's environment, including also information from family caregivers.

In present study, we review significant VR in clinical neuroscience practice in diagnosis and therapy/rehabilitation for elderly people and patients with neurological disorders and/or motor disability, such as multiple sclerosis, muscular dystrophy, peripheral neuropathy, stroke, amyotrophic lateral sclerosis, Parkinson's and Alzheimer's patients. Clinical professionals are looking for novel/innovative technologies to help patients on their path to better functioning in their daily live. Many findings suggest that VR in therapy/rehabilitation can be more motivating and effective for patients than a traditional approach. Thus, the newest VR and bioinformatics technologies allow a new way of providing traditional healthcare and are valuable and helpful tools to diagnose/recognize (fatal/genetic) diseases and manipulative therapy in rehabilitation of cognitive and locomotor systems.

\section{Novel perspective of artificial intelligence and machine learning in medical and genetic practice}

\section{Beata Sokołowska}

Bioinformatics Laboratory, Mossakowski Medical Research Centre Polish Academy of Sciences, Warsaw, Poland

Machine learning $(\mathrm{ML})$ is a scientific discipline in the intersection of computer science/informatics, statistics, and applied mathematics. ML has developed into one of the main pillars of modern artificial intelligence (Al) as well as the emerging research area of data science (i.e. sensing, computation and communication technologies). Al-ML in medicine may be described as the scientific field related to research studies, projects, and applications that aim to support decision-based (bio)medical tasks through knowledge or data computer-based solutions.

Novel Al-ML algorithms are used in following the important applications in medical research and clinical practice, including in particular (neuro)pathological and (neuro)genetic data, for (1) innovative diagnostics, (2) new drug development faster, (3) therapy/treatment personalization, and (4) gene editing. DIAGNOSTICS: new Al-ML approaches involve heterogeneous data sources such as CT/MRI, genomics/proteomics/ metabolomics and patient databases in assessing diseases and/or their progression. The innovative diagnostic systems have recently made huge advances in automatically diagnosing diseases, making diagnostics effective and more accessible. DEVELOP DRUGS: novel AI-ML have already been used widely and successfully in main stages of drug development such as: (a) identifying targets for intervention; (b) discovering drug candidates; (c) speeding up clinical trials; (d) finding markers for diagnosing the disease (i.e. diagnostic, prognostic and predictive biomarkers).

Personalize therapy/treatment: patients can respond to medications and medical procedures differently, and Al-ML can automate this complicated statistical analysis - and help discover which features indicate that a patient will have a particular response to therapy/treatment undertaken. So the algorithms can predict a patient's probable response to a treatment. The systems learn this by cross-referencing similar patients and comparing their treatments and outcomes.

Improve gene editing: Al-ML algorithms/models have been proven to produce the best results when it comes to predicting the degree of both guide-target interactions and off-target effects for a given (complex) RNA, and in perspective development of guide RNA for every region of human DNA.

Future perspectives: Computational intelligence, as well as intelligent devices/instruments and computational platforms/models can be useful to find valuable formulas/pat- 
terns and use them to make accurate, cost-effective decisions in complex conditions and analytical processes in human biology, healthcare, medicine and clinical practice.

\section{Neurogenetic diseases in "Digital brain" collection}

Tomasz Stępień ${ }^{1}$, Maciej Golan ${ }^{1}$, Albert Acewicz ${ }^{1}$, Dominik Chutorański ${ }^{1}$, Sylwia Tarka ${ }^{1,2}$, Anna Mietelska-Porowska ${ }^{1,3}$, Paulina Felczak ${ }^{1}$, Natalia Chmura ${ }^{1}$, Ludomir Z. Niewiadomski ${ }^{1}$, Teresa Wierzba-Bobrowicz ${ }^{1}$

${ }^{1}$ Department of Neuropathology, Institute of Psychiatry and Neurology, Warsaw, Poland

2Department of Forensic Medicine, Medical University of Warsaw, Poland

${ }^{3}$ Neurobiology Center, Nencki Institute of Experimental Biology, Polish Academy of Science, Warsaw, Poland

Poland has one of the largest brain collections in the world, in the Department of Neuropathology at the Institute of Psychiatry and Neurology in Warsaw. For over half a century, subsequent generations of neuropathologists created the archive that currently forms the First Polish Brain Bank. The collection includes patients' brains containing examples of many diseases. Transferal of this brain archive onto a digital platform will enable wider access to the whole catalogue of the Institute of Psychiatry and Neurology including: photographs of whole brain slices, paraffin-embedded brain fragments, microscopic scans of histological preparations of brain sections, as well as clinical and neuropathological diagnoses.

The project "Digital brain - digital collection of the Institute of Psychiatry and Neurology in Warsaw" (Project No. POPC.02.03.01-00.0042/18) meets the conditions regarding the development of Polish science, among others, searching the genetic connections with the occurrence of disorders in the central and peripheral nervous system. Among many disease entities collected in the First Polish Brain Bank the genetic diseases include: familial Alzheimer's disease (fAD, chromosome 14q24), fronto-temporal lobe degeneration with dementia (FTLD, IVS10 + 3G>A MAPT), progressive supranuclear palsy (Steele-Richardson-Olszewski disease) (chromosome 17, MAP-tau gene), lateral amyotrophic sclerosis (SLA, chromosome 21922), autosomal dominant variants of cerebellar ataxia (ADCA) SCA1 and SCA2 (chromosome 6q23, chromosome 12q24), Down syndrome (trisomy of chromosome 21), Krabbe syndrome leukodystrophy (chromosome 14q31), adrenoleukodystrophy (chromosome Xq28), GM1 gangliosidosis (GLB1), GM2 gangliosidosis (chromosome 5q13), cerebral amyloid angiopathy (polymorphism of $A p o E$ gene), Huntington's disease (HD, chromosome 4p16). By use of advanced molecular techniques, the resources of the First Polish Brain Bank will enable retrospective characterisation of rare, and difficult to treat, genetically-determined diseases.

Moreover, this unique collection can be used for scientific research, statistical and popular science research, as well as a teaching base for promoting knowledge in the field of neuropathology, neurology, neuro-oncology and psychiatry. In addition, use of the First Polish Brain Bank will help expand knowledge regarding the pathological mechanisms of diseases, support the medical and scientific programs seeking new therapeutic strategies and assist the development of new methods of treatment and rehabilitation of patients with mental, neurological and neuro-oncological disorders.

\section{Dynamic mutations in genetic diagnostics}

\section{Anna Sułek}

Department of Genetics, Institute of Psychiatry and Neurology, Warsaw, Poland

Dynamic mutations causing multiplication of microsatellite repeats leads to many degenerative diseases of the central nervous system. These include: Huntington's disease (HD), spinocerebellar ataxia (SCA), spinobulbar muscular atrophy (SBMA), fragile $X$ syndrome and myotonic dystrophies (DM1 and DM2). The most common are trinucleotide repeats, which can be located both in the coding parts of genes (HD, SCA 1, 2, 3, 6, 7, 17, SBMA) and in non-translated regions (FRAX, DM, SCA8, SCA12).

For each of the genes, a specific range of repeats was established in which the microsatellite number is stable and does not tend to expand. It usually does not exceed 40 repeats. However, alleles with a large but still normal number of repeats may lose stability and expand, which is synonymous with de novo mutation. Expansion of the repeats number and exceeding this normal range underlies the so-called dynamic mutations and disease process. Molecular diagnostics of diseases caused by dynamic mutations consists in DNA analysis and determining the number of microsatellite repeats in the genes associated with the occurrence of the specific disorders mentioned above. For microsatellite repeats located in non-coding regions of the gene, the observed level of instability can be much greater and dynamic mutations can lead to expansion of several hundred or even several thousand repeats.

In diseases with autosomal dominant inheritance, the risk of transmission of the abnormal allele of a sick parent 
to the children is $50 \%$. The use of molecular research in the diagnosis of neurodegenerative diseases is of great importance for genetic counseling. The patient and his family can be offered the following types of tests:

- Diagnostic test - search for dynamic mutations in genes related to the occurrence of clinical disease symptoms;

- Presymptomatic test - performed at the request of adults from a family in which the diagnosis of the disease was established based on molecular tests in the proband;

- Prenatal testing - fetal DNA analysis if one parent has previously had an expansion of microsatellite replicates.

\section{Central nervous system defects and genetics}

Krzysztof Szczałuba ${ }^{1}$, Krystyna Szymańska², Milena Laure-Kamionowska ${ }^{2}$, Rafał Płoski ${ }^{1}$

1Department of Medical Genetics, Warsaw Medical University, Poland

2Department of Clinical and Experimental Neuropathology, Mossakowski Medical Research Centre, Polish Academy of Sciences, Warsaw, Poland

Central nervous system (CNS) defects occur with an incidence of around 15/10,000 live births and are isolated in approximately $80 \%$ of cases. The remaining $20 \%$ of CNS defects occur in a syndromic form (together with other developmental anomalies and/or dysmorphic features).

From an embryological point of view, the causative agent of CNS defects can work at each of the five stages of development. In the first - neurulation - possible damage leads to the formation of dysraphic defects of the spinal cord and canal, and less often of the brain and skull, which results in a hernia of the posterior (meningocele) and cephalic (cerebral) segment, respectively. In the second stage, the structure of the brain follicles is disturbed, and the basic example is the imperfect division of the forebrain (holoprosencephaly). The other three stages of CNS development concern the cerebral cortex. Nerve cell proliferative disorders include, but are not limited to, true microcephaly caused by cell spindle dysfunction. The key role in early neuronal migration disorders is played by filamine $\mathrm{A}$ and the FMR1 gene. Late migration disorders are characterized by abnormal shaping (organization) of the cerebral cortex, which, among other things, has neuro-radiological and clinical effects in the form of lissencephaly and polymicrogyria.

The presentation will show different examples of genetically determined CNS defects and clinical guidelines for their diagnosis and therapeutic management.

\section{VGVAPG peptide increase proliferation of mouse cortical astrocyte in vitro}

\author{
Konrad A. Szychowski, Klaudia Dachowska, \\ Jakub Tobiasz, Jan Gmiński
}

Department of Public Health, Dietetics and Lifestyle Disorders, Faculty of Medicine, University of Information Technology and Management in Rzeszow, Poland

Degradation products of elastin, elastin-derived peptides (EDPs) are detectable in cerebrospinal fluid of both group of healthy subjects and patients with ischemic and hemorrhage stroke. To date, it has been well described that main effects of EDPs are mediated through the interaction with an elastin binding protein (EBP), identified as an enzymatically inactive spliced variant of Glb1 gene. Number of papers shows that EDPs or conservative sequence repeated in elastin molecules (VGVAPG) increase proliferation of fibroblasts, monocytes and different cancer cell lines. However, previous studies of our team show that VGVAPG peptide decrease proliferation of human neuroblastoma cell line. Therefore, the aim of this research was to investigate the impact of VGVAPG peptide on proliferation in mouse astrocyte in vitro. The cultures of cortical astrocytes were prepared from Swiss mouse embryos on 17/18 days of gestation. The cells were cultured in phenol red-free DMEM/F12 medium supplemented with 1\% and $10 \%$ FBS and were exposed to increasing concentrations of VGVAPG peptide for 48 and $72 \mathrm{~h}$. Moreover, siRNA gene silencing technique was applied. Afterwards, mRNA was collected and gene expression was measured by qPCR method and resazurine reduction assay. The results showed that after 48 and $72 \mathrm{~h}$ of exposure to VGVAPG peptide increase proliferation of astrocyte. Proliferation rate depend on level of FBS. Our data suggests that effects caused by VGVAPG peptide depend on EBP. However, more research underlying mechanism of VGVAPG peptide action in nervous system is needed.

Supported by University of Information Technology and Management in Rzeszow (DS. 503-07-01-08).

\section{Metabolic and genetic disorders mimicking cerebral palsy}

Krystyna Szymańskaํ, Krzysztof Szczałuba², Milena Laure-Kamionowska ${ }^{1}$, Rafał Płoski ${ }^{2}$
${ }^{1}$ Department of Clinical and Experimental Neuropathology, Mossakowski Medical Research Centre, Polish Academy of Sciences, Warsaw, Poland
${ }^{2}$ Department of Medical Genetics, Medical University of Warsaw, Poland

Cerebral palsy (CP) is an umbrella term which describes a group of "permanent, non-progressive disorders that 
affect muscle tone, posture, and movement due to abnormalities of the developing fetal or infantile brain resulting from a variety of causes".

Some genetic conditions with a slowly progressive or non-progressive course may mimic the clinical manifestations of CP and must not be misdiagnosed. Recent genetic studies of CP patients using whole exome sequencing (WES) show that $14 \%$ of cases have single-gene mutations. The authors discuss the use of the term cerebral palsy in reference to the group of patients with a genetic disease.

\section{Neuropathological presentation of the child with POLG gene}

\author{
Sylwia Tarka1,2, Teresa Wierzba-Bobrowicz², \\ Milena Laure-Kamionowska ${ }^{3}$, Katarzyna Witulska ${ }^{4}$, \\ Elżbieta Ciara ${ }^{5}$, Paulina Felczak ${ }^{2}$, Tomasz Stępień ${ }^{2}$ \\ ${ }^{1}$ Departament of Forensic Medicine, Medical University \\ of Warsaw, Poland \\ ${ }^{2}$ Department of Neuropathology, Institute of Psychiatry \\ and Neurology, Warsaw, Poland \\ ${ }^{3}$ Department of Experimental and Clinical Neuropathology, \\ Mossakowski Medical Research Centre, Polish Academy \\ of Sciences, Warsaw, Poland \\ ${ }^{4}$ Department of Intensive Care, Children's Memorial Health \\ Institute (CMHI), Warsaw, Poland \\ ${ }^{5}$ Department of Medical Genetics, Children's Memorial Health \\ Institute (CMHI), Warsaw, Poland
}

8-years old girl with epilepsia and mild intellectual disability, after 2 months of valproic acid (VPA) treatment, was admitted to the hospital, complaining of severe abdominal pain and vomiting. A diagnosis of acute pancreatitis was made at the beginning, then appendicitis as well as acute hepatic insufficiency of unknown etiology (including Wilson's disease) were suspected. The patient's condition deteriorated very quickly. Four days after onset of the abdominal symptoms respiratory and circulatory failure as well as irreversible liver damage was diagnosed and the girl was qualified for the liver transplantation. A thorough analysis of the entire course of the disease has allowed to suspect the mitochondrial disease and material for genetic diagnosis was collected. The child died on the sixth day after the onset of abdominal symptoms. General autopsy, pathomorphological and neuropathological examination was performed. Pathomorphological examination showed massive pancreatic necrosis with acute inflammatory infiltration, focal liver necrosis with small inflammatory infiltration and cholestasis.

Main findings in the neuropathological examination were: hypertrophic and deformed neurons and many multinuclear, bizarre astrocytes were find bilaterally in the medulla oblongata; degeneration of midbrain's substantia nigra, and pathological forms of astroglia in basal ganglia. Focal demyelination, accompanied by mild spongiosis was observed in the both hemispheres of brain and cerebellum.

Genetic testing results, obtained post mortem, revealed autosomal, recessive mutation in the POLG gene, resulting in a deficit of gamma polymerase. Summary of the genetic tests, neuropathological findings and clinical presentations allows to make a diagnosis of mitochondrial disease.

Acute liver failure induced by valproic acid administration in children with epilepsy due to mitochondrial disease is videly described in the literature, but acute pancreatitis is extremely rare. Clinical presentation of mitochondrial disease is heterogenous, and certain diagnosis requires genetic testing.

\section{The analysis of C9orf72 mutation in Huntington disease patients with excluded HTT dynamic mutation}

Filip Tomczuk ${ }^{1}$, Ewelina Elert-Dobkowska ${ }^{1}$, Wiktoria Radziwonik $^{1}$, Paulina Małczyńska ${ }^{1}$, Iwona Stępniak², Karolina Ziora-Jakutowicz ${ }^{2}$, Jacek Zaremba², Anna Sutek ${ }^{1}$

${ }^{1}$ Laboratory of Molecular Basis of Neurodegenerative Diseases, Department of Genetics, Institute of Psychiatry and Neurology, Warsaw, Poland

${ }^{2}$ Genetic Counseling Unit, Institute of Psychiatry and Neurology, Warsaw, Poland

Dynamic mutation act as an unstable heritable element where the probability of a mutant phenotype expression is a function of the number of the repeats within. These mutations, typically short sequences repeated many times, give rise to numerous known diseases.

A repeat expansion in the C9orf72 gene has recently been identified as a major cause of familial and sporadic frontotemporal lobar degeneration and amyotrophic lateral sclerosis (ALS). Moreover, Huntington disease-like (HDL) syndrome due to C9ORF72 expansions is observed as a rare, genetic neurodegenerative disease characterized by movement disorders, including dystonia, chorea, myoclonus, tremor and rigidity. Associated features are also cognitive and memory impairment, early psychiatric disturbances and behavioral problems.

In many cases where Huntington disease (HD) is suspected, the genetic test for HD is negative: these are known as HD phenocopies. In this study, we aim to assess the frequency of C9orf72 expansions in a cohort of HD phenocopies patients. The mutation of C9orf72 is a hexanucleotide repeat expansion of the six nucleotides (GGG- 
GCC $)_{n}$. In a healthy person, there are few repeats of this hexanucleotide, typically less than 20-30, but in people with the mutation, the repeat can occur in the order of hundreds.

A cohort of $1046 \mathrm{HD}$ patients with excluded HTT mutations where analyzed for the C9orf72 expansion using repeat primed PCR. We identified three cases that had hexanucleotide $(G G G G C C)_{n}$ repeat expansion within C9orf72 gene. In these patients the first symptoms appeared at a young age and mainly problems with muscle coordination and control.

These findings suggest that C9orf72 repeat expansion is a rare cause of clinical symptoms in patients with HTT repeat expansion excluded.

\section{Alzheimer's disease and diabetes mellitus: neuromorphology of cognitive impairment}

\author{
Liliya Volos ${ }^{1}$, Tatiana Mykhaylichenko ${ }^{2}$ \\ ${ }^{1}$ Department of Pathological Anatomy, Danylo Halytsky Lviv \\ National Medical University, Lviv, Ukraine \\ ${ }^{2}$ Department of Endocrinology, Donetsk National Medical \\ University, Lyman, Ukraine
}

Introduction: Interconnection between diabetes mellitus (DM) and Alzheimer's disease (AD) has recently been the subject of intense studying of experts. DM is associated with an increased risk of cognitive impairment, dementia and stroke. Patients with diabetes mellitus were twice as likely to develop AD - a neurodystrophic disease with progression of memory disorders and higher cortical functions. There are many common pathogenetic mechanisms underlying both diseases. Some researchers have supported the idea that AD can be considered as type 3 diabetes. However, a specific mechanism for the development of $A D$ on the background of high glucose concentration has not been established. Separate studies have linked type 2 diabetes with Alzheimer's type dementia. In spite of the established links between type 2 diabetes and AD, the interrelated pathogenetic mechanisms of cognitive impairment have not been fully explored.

Aim of the study: To identify common neuromorphological changes in type 2 diabetes and AD that potentially affect cognitive impairment.

Material and methods: We have performed a clinical-morphological comparison of outpatient card data, disease histories and autopsy reports of 11 cases of type 2 diabetes and 15 observations of AD.

Results: Alzheimer's neurofibrils and Alzheimer's plaques are typical signs of AD. Their number varies widely depending on the area of brain studying, the stage of the disease and the duration of clinical symptoms. The main cyto-angioarchitectonic manifestations of diabetic brain damage are diffuse alteration of the basement membranes and vascular endothelium, capillary fibrosis and hyalinosis, pericyte proliferation, congophilic angiopathy accompanied by a sharp disruption on transcapillary transport. There is a combination of acute and chronic processes, reversible and irreversible changes in nerve cells: neuronal swelling, subtotal chromatolysis, karyopyknosis and cytoplasmic homogenization, satellite disease, pericarion enlightenment, tigrolysis, lysis of neurons with the formation of "shadow cells". In areas of chronic ischemia there are neurons or groups of neurons with morphological signs of Alzheimer's neurodegeneration (pathological neurofibrils in the form of tangles), a large number of hematoxylin spheres and single Lafora bodies. Moreover, single terminal plaques are found in the impregnation of silver by the Bielschowsky method.

Conclusions: Thus, the common brain morphological changes in type 2 diabetes mellitus and AD are formation neurofibrillary tangles are thought to contribute to the degradation of the neurons in the brain, congophilic angiopathy of small vessels and vessels of medium caliber. The combination of vascular and neurodegenerative components can mutually potentiate each other, causing clinical symptoms of cognitive deficits.

\section{Neuropathological changes in a child with a POLG mutations in Alpers-Huttenlocher syndrome}

\section{Liliya Volos ${ }^{1}$, Mariya Dushar², Akopyan Hayane ${ }^{2,3}$, Olya Kovalyk ${ }^{4}$, Iryna Loshak ${ }^{5}$, Galyna Gubych ${ }^{5}$}

${ }^{1}$ Danylo Halytsky Lviv National Medical University, Lviv, Ukraine ${ }^{2}$ SI "Institute of Hereditary Pathology, NAMS of Ukraine", Lviv, Ukraine

${ }^{3}$ Faculty of Medicine, Rzeszow University, Poland

${ }^{4}$ Lviv Pathological and Anatomical Bureau, Ukraine

${ }^{5}$ Western-Ukrainian Specialized Children's Medical Centre, Lviv, Ukraine

Introduction: Alpers-Huttenlocher syndrome is a rare mitochondrial disease most often associated with mutations in the mitochondrial DNA replicase, polymerase gamma. The hallmark clinical features of Alpers-Huttenlocher syndrome are intractable seizures, develop mental regression and liver dysfunction. The prognosis for patients with Alpers-Huttenlocher syndrome is poor. Common causes of death are fatal encephalopathy and liver failure.

Aim of the study: To identify and describe the case of an 8 month old baby with epilepsy and liver failure on the background of valproic acid treatment with a fatal case. 
Material and methods: The clinical-morphological data of the disease and the results of the histological study indicated the presence of Alpers-Huttenlocher syndrome in the 8 month old child. At the Institute of Mother and Child in Warsaw (Instytut Matki i Dziecka, Warszawa), molecular analysis of the POLG1 gene was carried out by next generation sequencing (NGS). The child's DNA was stored in the laboratory of the Lviv Institute of Hereditary Pathology. The authors thank the National Research Institute of Mother and Child in Warsaw for help in conducting a molecular genetic study of the Alpers-Huttenlocher syndrome.

Results: The diagnosis of Alpers-Huttenlocher syndrome was confirmed by molecular genetic study post mortem. The primary organs in our case of Alpers-Huttenlocher syndrome demonstrating histologic changes were the liver and brain. Neural tissue of the cerebral cortex showed spongiosis, neuronal loss, and astrocytosis. The hepatic changes were specific to Alpers-Huttenlocher syndrome. It was the bridging fibrosis, bile ductular proliferation, collapse of liver plate cells, hepatocyte dropout or focal necrosis, microvesicular steatosis, oncocytic change, regenerative nodules, and disorganization of the normal lobular architecture.

Two heterozygous variants of the POLG1 gene were identified, one of which: p 1399G>A, p. (Ala467Thr) is well known and described in the bases of human mutations as a recessive pathogenic variant, as well as the second: p.1242_1244delCTT, p. (Phe414del) is a potentially pathogenic recessive variant, which has been described in the literature in one patient with mitochondrial encephalopathy. This pathology is one of the diseases caused by a mutation in the POLG1 gene. To determine the bioavailability of these changes, a genetic study was conducted on the parents of the child, who found that each of them had one mutation. Thus, confirmed Alpers-Huttenlocher disease in a child.

Conclusions: Triad of clinical hepatocerebral symptoms and seizures and molecular analysis of the POLG1 gene constitute the clinical diagnosis of Alpers-Huttenlocher syndrome.

\section{TDP43 protein in cerebral amyloid angiopathy in $A P O E$ heterozygotes}

\section{Teresa Wierzba-Bobrowicz ${ }^{1}$, Tadeusz Mendel ${ }^{2}$, Dominik Chutorański ${ }^{1}$, Magdalena Konarzewska ${ }^{3}$, Grażyna Kostrzewa ${ }^{3}$, Tomasz Stępień ${ }^{1}$, Albert Acewicz ${ }^{3}$, Sylwia Tarka ${ }^{1,3}$, Paulina Felczak ${ }^{1}$}

${ }^{1}$ Department of Neuropathology, Institute Psychiatry and Neurology, Warsaw, Poland

${ }^{2}$ Department of Neurology, Institute Psychiatry and Neurology, Warsaw, Poland
${ }^{3}$ Department of Forensic Medicine, Medical University of Warsaw, Poland

Transactive response DNA-binding protein 43 (TDP-43) is a protein that in humans is encoded by the TAR DBP gene. TDP-43 is expressed nuclear protein that binds both DNA and RNA, and is shuttling between nucleus and cytoplasm of neurons and neuroglial cells, regulating many aspects of RNA processing.

In aging and some neurodegenerative diseases including limbic-predominant age-related TDP-43 encephalopathy (LATE) the TDP-43 protein is aggregated in neurons and the glial cells forming pathological inclusions.

It has been reported that some genes, including gen $A P O E$ contributes to the formation of these pathological aggregates of TDP43 protein.

Cerebral amyloid angiopathy (CAA) is characterized by cerebrovascular deposition of the amyloid $\beta$-peptide. The normal function of $\beta$-amyloid is not exactly known.

A strong relationship between the APOE $\varepsilon-3 / 4$ alleles and the presence of CAA were observed. The apoE is a polymorphic protein that plays a central role in metabolism of cholesterol and the triglycerides.

We tested, if in cases of CAA disease with APOE $\varepsilon-3 / 4$ (it was the most common), pathological deposites of TDP43 proteins were observed in the human brains.

In most of the CAA cases studied (18/20), the antiTDP-43 antibody recognized pathological inclusions in the nuclei and neuronal cytoplasm, neurites in vascular endothelial cells and astrocytes. 
